\title{
Scalar Mesons and Multichannel Amplitudes
}

\author{
R. Kamiński ${ }^{1}$, L. Leśniak ${ }^{1}$ and B. Loiseau ${ }^{2}$ \\ ${ }^{1}$ Department of Theoretical Physics \\ H. Niewodniczański Institute of Nuclear Physics, \\ PL 31-342 Kraków, Poland \\ ${ }^{2}$ LPTPE Université P. et M. Curie, 4, Place Jussieu, \\ 75252 Paris CEDEX 05, France
}

February 26, 2019

\begin{abstract}
Properties of scalar-isoscalar mesons are analysed in an unitary model using separable interactions in three decay channels: $\pi \pi, K \bar{K}$ and an effective $2 \pi 2 \pi$. We obtain different solutions by fitting various data on the $\pi \pi$ and $K \bar{K}$ phase shifts and inelasticities including the CERN-Cracow-Munich measurements of the $\pi^{-} p_{\uparrow} \rightarrow \pi^{+} \pi^{-} n$ reaction on a polarized target. Analytical structure of the meson-meson multichannel amplitudes is studied with a special emphasis on the role played by the $S$-matrix zeroes. $S$-matrix poles, located in the complex energy plane not too far from the physical region, are interpreted as scalar resonances. We see a wide $f_{0}(500)$, a narrow $f_{0}(980)$ and a relatively narrow $f_{0}(1400)$. In one of our solutions a resonance at about $1700 \mathrm{MeV}$ is also found. Total, elastic and inelastic channel cross sections, branching ratios and coupling constants are evaluated and compared with available data. We construct an approximation to our model and show that the Breit-Wigner approach has a limited phenomenological applicability.
\end{abstract}

\section{Introduction}

Full classification and identification of scalar mesons is not yet well established [1]. Many theoretical and experimental efforts have been recently made for a better understanding of the scalar mesons as it can be seen, for example, in references given in [1, 2]. From QCD one expects presence of some scalar $\left(J^{P C}=0^{++}, I=0\right)$ glueballs which can be mixed with ordinary $q \bar{q}$ scalar states [3]. The lowest scalar glueball masses predicted by lattice QCD calculations are in the range $1500 \mathrm{MeV}$ to $1700 \mathrm{MeV}[$, 5]. There are now lively discussions about the nature of scalar mesons 
$f_{0}(1500)$ and $f_{J}(1710)$ and their possible mixture with scalar glueballs [1, 6]. High statistics experiments on meson productions such as $p \bar{p}$ annihilation, $\pi N$ scattering on unpolarized and polarized targets, central production in $p p$ scattering, $J / \Psi$ or other heavy meson decays and $\gamma \gamma$ collisions have been performed. Their analyses give some new evidence for the existence of five scalar-isoscalar mesons $f_{0}(400-1200)$ or $\sigma, f_{0}(980), f_{0}(1370), f_{0}(1500)$ and $f_{0}(1710)$ [1].

In [7] we have analysed new solutions for the scalar-isoscalar $\pi \pi$ phase shifts [8] together with previous $K \bar{K}$ results in the framework of a three coupled channel model based on an extension of the two-channel model of [9]. Separable potentials were used to describe interactions in the $\pi \pi, K \bar{K}$ and an effective $2 \pi 2 \pi$ channels. This third channel was introduced to take into account a strong four-pion production observed in different experiments. In these data some evidence for four pion clustering into $\sigma \sigma$ pair, coming from strong interaction between two pions was found (see for example [10]). Our $2 \pi 2 \pi$ effective channel, called $\sigma \sigma$, can however represent also other possible clusterings such as $\rho \rho, a_{1}(1260) \pi, \pi(1300) \pi$ and $\omega \omega$. One should not mix the effective threshold mass with twice the mass of the $f_{0}(500)$ or $\sigma$ resonance which is seen in the $\pi \pi$ channel.

The parameters of the model were determined by a fit to two sets of $\pi \pi$ phaseshifts and inelasticities obtained in a recent analysis of the CERN- Cracow-Munich measurements of the $\pi^{-} p_{\uparrow} \longrightarrow \pi^{+} \pi^{-} n$ reaction on a polarized target [8]. It was stressed in [8] that the $a_{1}$ exchange gives an important contribution to the $\pi^{-} p \rightarrow$ $\pi^{+} \pi^{-} n$ reaction amplitudes. Recently Achasov and Shestakov have also come to the conclusion that the $a_{1}$ exchange plays an important role in the reaction $\pi^{-} p \rightarrow$ $\pi^{0} \pi^{0} n$ on unpolarized target [1]. This conclusion may be experimentally verified by measurements on polarized target. The $\pi \pi$ data [8], covering the 600 to $1600 \mathrm{MeV}$ $\pi \pi$ invariant mass range, were completed in the lower energy region by the $\pi \pi$ phase shifts of [12, 13] and [14]. Further constraints were imposed by using the $K \bar{K}$ phase shifts from the $K \bar{K}$ threshold up to $1530 \mathrm{MeV}$ [15].

We found a relatively narrow $(90-180 \mathrm{MeV})$ scalar resonance $f_{0}(1400-1460)$. Our analysis of previous CERN-Munich unpolarized target data [16] predicted a much broader $(\Gamma \approx 500 \mathrm{MeV})$ state. We have also obtained a very wide $(\Gamma \approx 500$ $\mathrm{MeV}) f_{0}(500)$ resonance and the well established narrow $f_{0}(980)(\Gamma \approx 60-70 \mathrm{MeV})$. Our model allows a theoretical study on the origin of resonances by switching off the interchannel couplings. In all the solutions found in [7] the $K \bar{K}$ interaction was repulsive or not attractive enough to create, by itself, a $K \bar{K}$ bound state.

In this paper we built two new solutions in which the $K \bar{K}$ state is bound in the uncoupled case. Our solutions are also characterized by presence or absence of a $\sigma \sigma$ bound state. Gradual increase of interchannel couplings allows one to link the Smatrix poles from the uncoupled to fully coupled case. In our approach experimental resonances correspond to the S-matrix poles in the complex energy plane close to the physical region. This is in contrast to some other descriptions where resonance parameters are introduced using K-matrix poles or Breit-Wigner formulae with some ad-hoc background in each channel. Let us here remark that the Particle Data Group 
[1] has misplaced the $\sigma$ meson parameters found in [9]. They have been referred to under the name" $f_{0}(400-1200)$ Breit-Wigner mass or K-matrix pole parameters" on page 363 of [1] instead of being referred under the appropriate title " $f_{0}(400-1200)$ T-matrix pole $\sqrt{s}$ ". Similar misplacements were also made on pages 392 and 393 for the $f_{0}(1370)$. Studying the analytical structure of multichannel amplitudes we shall show the important role played not only by poles but also by zeroes of the Smatrix. Knowledge of the poles and zeroes enables us to give in some cases a simple phenomenological approximation of the T-matrix. Our results on scalar-isoscalar resonances, masses, widths, branching ratios, coupling constants as well as phase shifts, inelasticities and cross sections in the three channels will be discussed for our different solutions and compared with the available data.

In Section 2 we describe the two new solutions in addition to those previously presented in [7]. Section 3 contains an analysis of positions of the $S$-matrix poles in the three channel amplitudes. Section 4 describes the influence of the $S$-matrix poles and zeroes on phase shifts and inelasticities near the $f_{0}(1400)$ resonance. In Section 5 we comment on the limited applicability of Breit-Wigner approach in the multichannel meson scattering. Evaluation of branching ratios in two and three coupled channels is presented in Section 6 and coupling constants are discussed in Section 7. In Section 8 we present phenomenological parametrizations of multichannel amplitudes. Summary and conclusions are given in Section 9. In Appendix A the full formula for the Jost function of our three-channel model is supplied. Approximated formulae for the pion-pion $S$-matrix element, especially useful in vicinity of the $f_{0}(1400)$ resonance, are given in Appendix B.

\section{New and former solutions}

In [7] we have briefly presented our three-channel model of meson-meson scattering. This model describes simultaneously nine reactions in a unitary way. In addition to the $\pi \pi$ and $K \bar{K}$ channels an effective $\sigma \sigma$ channel is introduced in order to describe important $4 \pi$ production and rescattering processes. Here we use exactly the same notation and definitions as in [7]. We furthermore give, in Appendix A, the full formula for the Jost function $D\left(k_{1}, k_{2}, k_{3}\right)$. Its knowledge is sufficient to construct all the $S$-matrix elements and all the physical quantities which will be discussed in this paper. In [7] we have obtained four three-channel solutions called A, B, C and D based on $\chi^{2}$ fits to the $\pi \pi S$-wave isoscalar phase shifts and inelasticities, and to the $K \bar{K}$ phase shifts. If the interchannel couplings are switched off then the $K \bar{K}$ pair remains unbound in all four fits. In solutions $\mathrm{B}, \mathrm{C}$ and $\mathrm{D}$ the $K \bar{K}$ potential is repulsive, so a $K \bar{K}$ bound state cannot be formed. In the fit $\mathrm{A}$ the $K \bar{K}$ potential is attractive but its strength is too small to form a bound state. Parameters of the $K \bar{K}$ interactions, found in [7], were different from the $K \bar{K}$ parameters obtained in [9] where a $K \bar{K}$ bound system could be formed in absence of the interchannel interactions. We should, however, remember that in [9] a different 
$\pi \pi$ phase-shift solution, obtained from the data analysis on an unpolarized target by the CERN-Munich group [16], was used.

In [7] we have used several sets of the $\pi \pi$ phase shifts obtained in [8] from the data taken on a polarized target by the CERN-Cracow-Munich collaboration [17]. Now, one can ask if the data of [8] rule out any set of potential parameters leading to a $K \bar{K}$ bound state. We have therefore repeated the $\chi^{2}$ fits performed in [7] for the "down-flat" $\pi \pi$ solution by adding an additional constraint, namely that the $K \bar{K}$ potential is sufficiently strong to obtain a bound state in the uncoupled case. As a result of these studies we have obtained two new solutions $\mathrm{E}$ and $\mathrm{F}$ which will be described below. Similar studies could be done for the "up-flat" solutions C and $\mathrm{D}$ which have less good $\chi^{2}$ values as seen in Table 2 of [7].

Table 1: Separable potential parameters for the solutions E and F. Values of $\beta$ and $m_{3}$ are given in $\mathrm{GeV}$.

\begin{tabular}{|l|c|c|}
\hline parameter & Solution E & Solution F \\
\hline$\Lambda_{11,1}$ & $-.26349 \times 10^{-3}$ & $-.13678 \times 10^{-3}$ \\
$\Lambda_{11,2}$ & -.18316 & -.17845 \\
$\Lambda_{22}$ & -.60400 & -.52087 \\
$\Lambda_{33}$ & $.17703 \times 10^{2}$ & -.73962 \\
$\Lambda_{12,1}$ & $.28776 \times 10^{-4}$ & $.89713 \times 10^{-6}$ \\
$\Lambda_{12,2}$ & .036838 & .048444 \\
$\Lambda_{13,1}$ & $-.34811 \times 10^{-3}$ & $-.14046 \times 10^{-3}$ \\
$\Lambda_{13,2}$ & .55929 & .095244 \\
$\Lambda_{23}$ & -1.5951 & -.024116 \\
$\beta_{1,1}$ & $.16355 \times 10^{4}$ & $.31518 \times 10^{4}$ \\
$\beta_{1,2}$ & 1.1052 & 1.0712 \\
$\beta_{2}$ & 1.4960 & 2.1224 \\
$\beta_{3}$ & .092701 & 1.4958 \\
$m_{3}$ & .675 & .680 \\
\hline
\end{tabular}

The resulting potential parameters for the solutions $\mathrm{E}$ and $\mathrm{F}$ are presented in Table 1. For the solution E we can notice a particularly strong repulsive coupling constant $\Lambda_{33}$ in the $\sigma \sigma$ channel. So the $\sigma \sigma$ interaction for the solution E will not form any bound state when the interchannel couplings are set equal to zero. This is in contrast to the solution $\mathrm{B}$ where such a state exists. The $\sigma \sigma$ bound state is also present in the solution $\mathrm{F}$ which has in addition a bound $K \bar{K}$ state. In Table 2 we give the binding properties when couplings between channels are switched off and the different $\chi^{2}$ values of the four solutions $\mathrm{A}, \mathrm{B}, \mathrm{E}$ and $\mathrm{F}$. The $\chi^{2}$ values for the solution $\mathrm{F}$ are not as good as for the solutions A and B but they are still quite reasonable. The $\pi \pi$ phase shifts of solution $\mathrm{F}$ increase by almost $180^{\circ}$ at $E \approx 1350 \mathrm{MeV}$ since at this energy a very narrow resonance $(\Gamma \approx 0.5 \mathrm{MeV})$ is 
Table 2: Comparison of four solutions fitted to the "down-flat" data of [8]. Second and third rows indicate presence or absence of bound states in $K \bar{K}$ and $\sigma \sigma$ channels when interchannel couplings are switched off. In the remaining rows the different $\chi^{2}$ values are specified. Numbers of experimental points are indicated in parenthesis. The $\overline{\chi^{2}}$ values result from fitting with reduced $\eta$ errors as explained in [7].

\begin{tabular}{|c|c|c|c|c|}
\hline Solution & $\mathrm{A}$ & $\mathrm{B}$ & $\mathrm{E}$ & $\mathrm{F}$ \\
\hline bound $K \bar{K}$ & no & no & yes & yes \\
\hline bound $\sigma \sigma$ & no & yes & no & yes \\
\hline$\chi_{\pi}^{2}(65)$ & 63.0 & 61.2 & 64.8 & 65.4 \\
\hline$\chi_{\pi K}^{2}(21)$ & 15.9 & 9.7 & 17.7 & 24.7 \\
\hline$\chi_{\eta}^{2}(30)$ & 13.2 & 12.9 & 12.6 & 10.5 \\
\hline$\chi_{\text {tot }}^{2}(116)$ & 92.1 & 83.8 & 95.1 & 100.6 \\
\hline$\overline{\chi_{\eta}^{2}}(30)$ & 36.7 & 29.3 & 33.4 & 33.6 \\
\hline$\overline{\chi_{\text {tot }}^{2}}(116)$ & 115.6 & 100.1 & 115.9 & 123.7 \\
\hline
\end{tabular}

created about $10 \mathrm{MeV}$ below the $\sigma \sigma$ threshold. This resonance makes also very narrow structures in the energy dependence of the $\pi \pi$ inelasticity and phase shifts. They cannot be, however, uniquely confirmed by the existing data, so we tend to treat the solution $\mathrm{F}$ as an interesting but not well experimentally confirmed example of a phenomenological set of separable potential parameters. The $\chi^{2}$ numbers of the solution $\mathrm{E}$ are better than those of the solution $\mathrm{F}$. They are comparable to those of the solution A, however, the solution B has the best $\chi^{2}$. Therefore in the following mainly the three solutions A, B and E will be simultaneously discussed. We shall furthermore present new physical quantities like cross sections, branching ratios and coupling constants which have not been discussed in paper [0]].

In Fig. 1 we compare, for energies above $1350 \mathrm{MeV}(\sigma \sigma$ threshold $)$, the inelasticities for the solution $\mathrm{E}$ in the three channels. Both $\pi \pi$ and $K \bar{K}$ inelasticities show a minimum with a different depth near $1600 \mathrm{MeV}$ and $1650 \mathrm{MeV}$, respectively. The $\sigma \sigma$ inelasticity, however, has two minima at 1475 and $1675 \mathrm{MeV}$. Second minimum can be related to an additional scalar resonance at about $1700 \mathrm{MeV}\left(f_{0}(1710)\right)$. Appearance of this resonance in addition to $f_{0}(1400)$ is a unique feature of the solution E. No such state exists in solutions A and B as it will be shown in detail in the next chapter. As we can see in Fig. 2 this state does not produce any strong increase of the $\pi \pi$ phase shifts which is often expected in the vicinity of a resonance. This fact will be explained in the next section.

In Fig. 3a,b we show inelasticities and phase shifts in the $\sigma \sigma$ channel for three solutions A, B and E. Lack of resonances above $1600 \mathrm{MeV}$ for the solutions A and B is responsible for a smooth energy dependence of the corresponding $\sigma \sigma$ inelasticities 


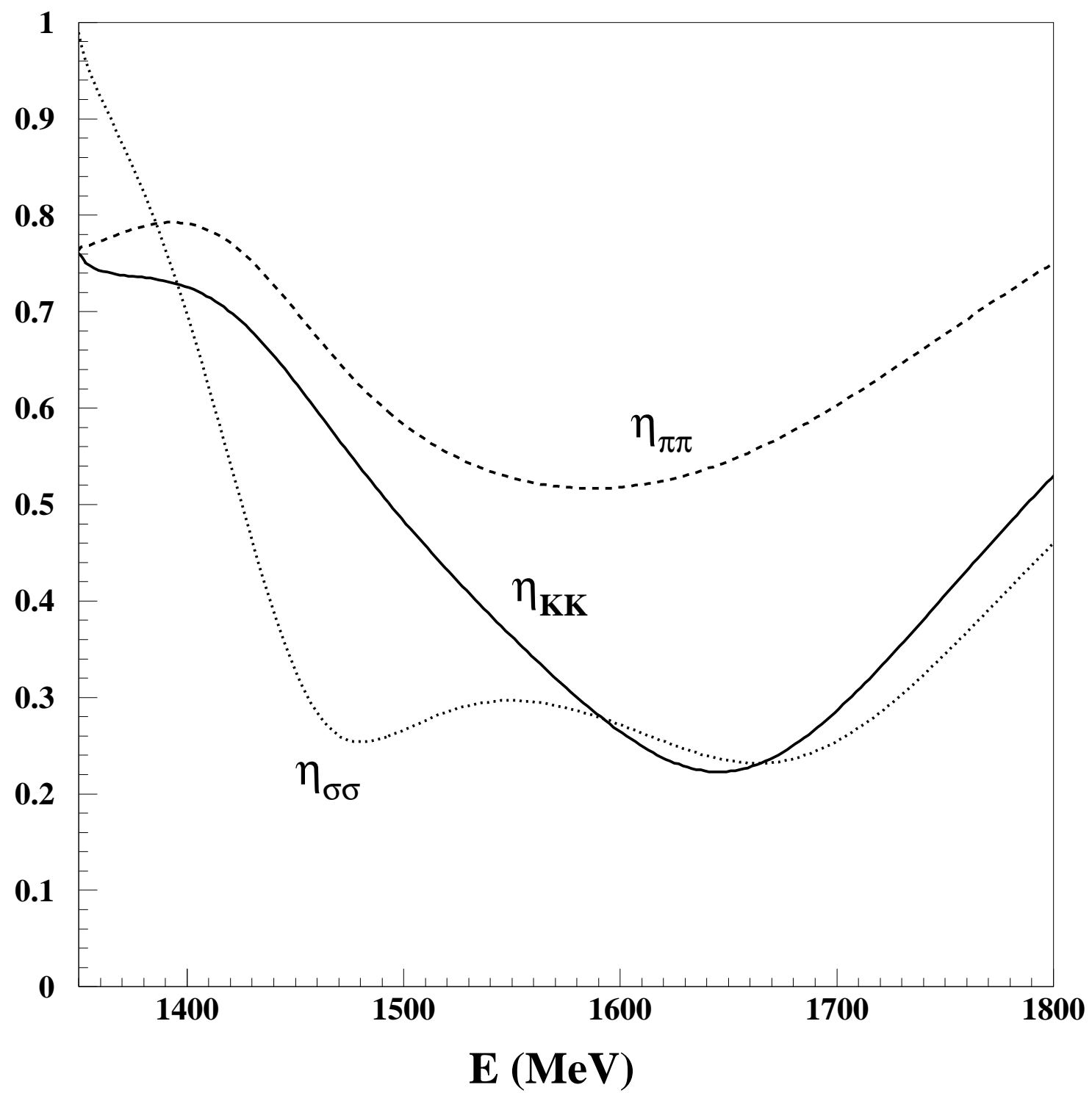

Figure 1: Energy dependence of inelasticity in the $\pi \pi, K \bar{K}$ and $\sigma \sigma$ channels for the solution E 


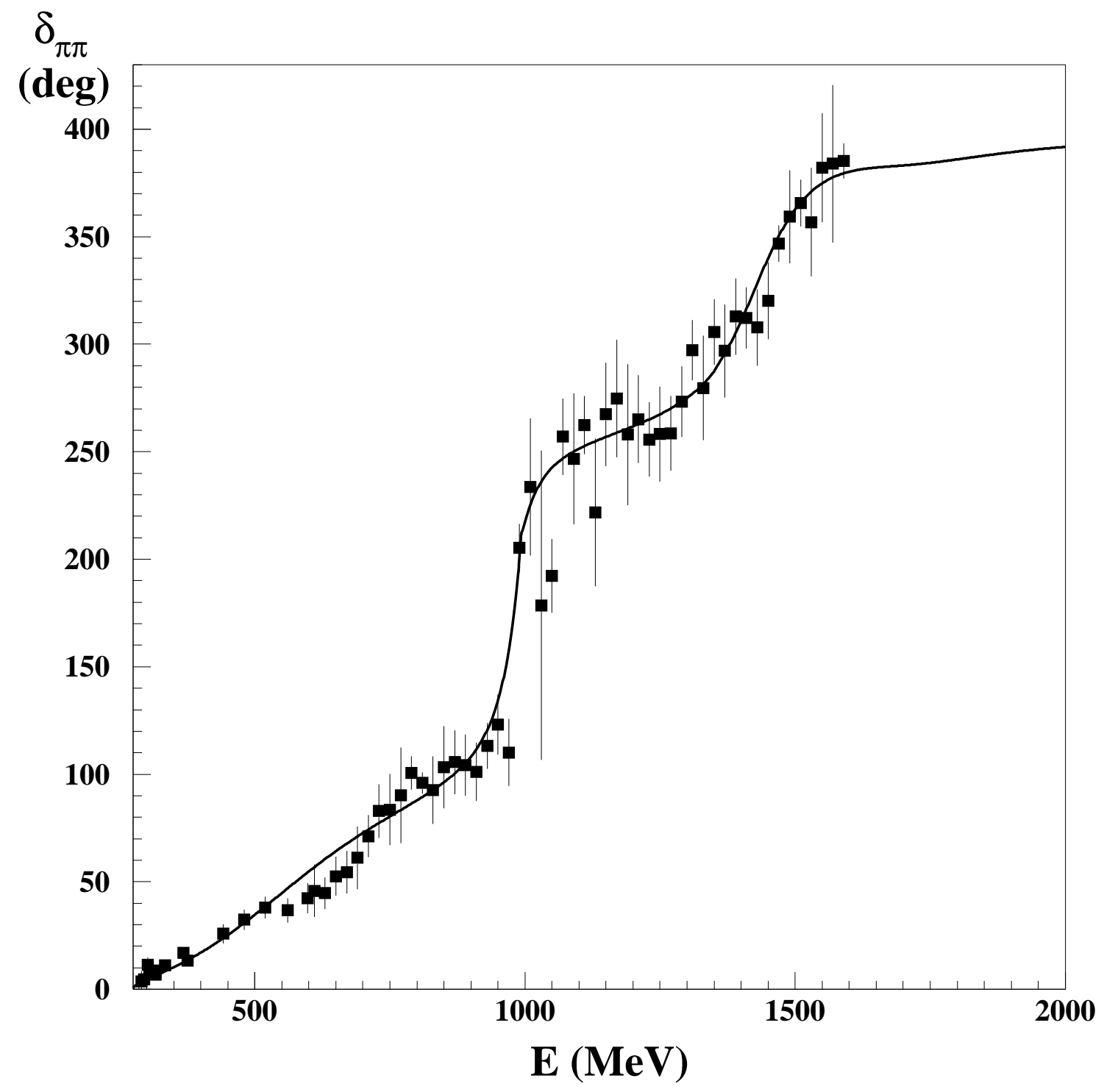

Figure 2: Energy dependence of $\pi \pi$ phase shifts for the solution E 

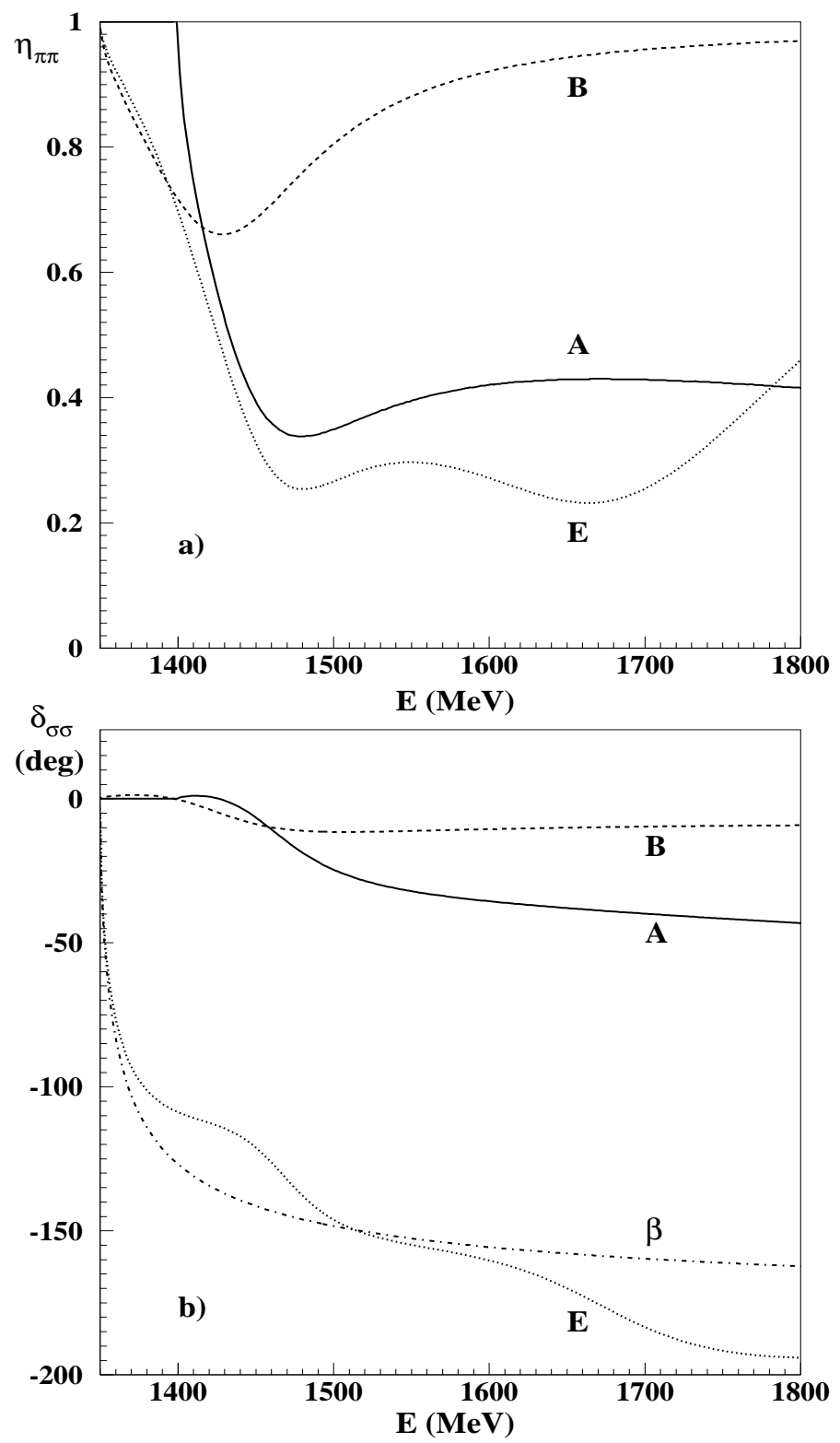

Figure 3: Energy dependence for the solutions A, B and $\mathrm{E}$ of $\sigma \sigma$ inelasticities a) and $\sigma \sigma$ phase shifts $\mathbf{b}$ ). The curve denoted by $\beta$ is the contribution of the double pole at $k_{3}=-i \beta_{3}$ for the solution $\mathrm{E}$. 
above $1500 \mathrm{MeV}$. A smooth decrease is also visible in the $\sigma \sigma$ phase shifts above 1450 $\mathrm{MeV}$. Influence of the $f_{0}(1400)$ resonance on the $\sigma \sigma$ phase shifts can be seen for the solutions $\mathrm{A}$ and $\mathrm{B}$ as a small maximum around 1450 and $1400 \mathrm{MeV}$, respectively. In the solution E a monotonous decrease is disturbed by two structures around 1425 and $1625 \mathrm{MeV}$ related to the $f_{0}(1400)$ and $f_{0}(1710)$ states, respectively. The " $\beta$ " curve in Fig. 3b will be discussed in the next section.

\section{Positions of poles}

A knowledge of the positions of the $S$-matrix poles in the complex energy plane is important for a physical interpretation of resonances. Some information about the positions of poles was already given in [7] for four solutions: A and B corresponding to the "down-flat" data of [8] and C and D corresponding to the "up-flat" data. Now we shall discuss in more detail three sets of poles, all corresponding to the "downflat" data, namely the solutions A, B and E which were described in the previous section. We think that the general structure of the solutions $\mathrm{C}$ and $\mathrm{D}$ is similar to those of sets A or B. In all the solutions $S$-matrix poles appear on different sheets of the complex channel momenta $k_{1}, k_{2}, k_{3}$. The sheets can be classified according to the signs of $I m k_{1}, I m k_{2}, I m k_{3}$. For instance, the notation --+ means that $I m k_{1}<0, I m k_{2}<0$ and $I m k_{3}>0$. Positions of the most important poles for the resonances $f_{0}(500), f_{0}(980)$ and $f_{0}(1400)$ were given in Table 3 of [7]. The origin of the resonances can be studied by a gradual increase of the interchannel coupling constants. In this way, starting from a case where all interchannel couplings are equal to zero, we can arrive to the coupled case and obtain a trajectory linking the positions of a given pole from the fully uncoupled to the fully coupled case. The structure of poles for the various solutions is significantly different. Positions of different $S$-matrix poles without and with couplings between channels are precised in Tables 3 to 6 for solutions A, B, E and F, respectively. In the two last columns the pole-sheet specification and their labels are given.

Let us recall that a given pole in the uncoupled channel splits into four poles in the coupled channel case since coupling of a given channel to any other channel doubles the number of related poles. This is a consequence of the analytic structure of the Jost function $D\left(k_{1}, k_{2}, k_{3}\right)$ describing three coupled channels. Furthermore, one should remember that

$$
D\left(k_{1}, k_{2}, k_{3}\right)=D^{*}\left(-k_{1}^{*},-k_{2}^{*},-k_{3}^{*}\right)
$$

therefore to a given zero of $D\left(k_{1}, k_{2}, k_{3}\right)$ at $k_{1}, k_{2}, k_{3}$ there always exists a twin zero at $-k_{1}^{*},-k_{2}^{*},-k_{3}^{*}$. In the complex energy plane a pole and its twin are symmetric with respect to the imaginary energy axis. In an uncoupled channel case zeroes and poles of $S$-matrix lie symmetrically with respect to the real energy axis. This symmetry is broken when the interchannel couplings are switched on. Therefore asymmetry in the localization of the zeroes is crucial to understand the energy dependence of phase 
shifts and inelasticities in all the coupled channels. Now we shall discuss specific features of the different solutions.

\section{3a) Solution A}

For the solution A the $\pi \pi$ channel poles at $E=(658-i 607) \mathrm{MeV}$ and $E=$ $(1346-i 275) \mathrm{MeV}$ in the uncoupled case evolve differently when the interchannel couplings are switched on (see Table 3). The first pole leads to a set of poles related

Table 3: Positions of $S$-matrix poles for the solution A (in $\mathrm{MeV}$ )

\begin{tabular}{|c|c|c|c|c|c|c|}
\hline \multirow[t]{2}{*}{ channel } & \multicolumn{2}{|c|}{ no couplings } & \multicolumn{2}{|c|}{ with couplings } & \multirow{2}{*}{$\begin{array}{c}\text { Sign of } \\
\operatorname{Im} k_{\pi} \operatorname{Im} k_{K} \operatorname{Im} k_{\sigma}\end{array}$} & \multirow[b]{2}{*}{ No } \\
\hline & $R e E$ & $\operatorname{ImE}$ & $R e E$ & $\operatorname{ImE}$ & & \\
\hline \multirow{5}{*}{$\pi \pi$} & \multirow{5}{*}{658} & \multirow{5}{*}{-607} & 564 & -279 & --- & $\mathrm{I}$ \\
\hline & & & 518 & -261 & -++ & II \\
\hline & & & 211 & 0 & -+- & III \\
\hline & & & 532 & -315 & --+ & IV \\
\hline & & & 235 & 0 & ++- & $\mathrm{V}$ \\
\hline \multirow{4}{*}{$\pi \pi$} & \multirow{4}{*}{1346} & \multirow{4}{*}{-275} & 1405 & -74 & --- & VI \\
\hline & & & 1445 & -116 & -++ & VII \\
\hline & & & 1424 & -94 & -+- & VIII \\
\hline & & & 1456 & -47 & --+ & IX \\
\hline \multirow{5}{*}{$K \bar{K}$} & \multirow{5}{*}{881} & \multirow{5}{*}{-498} & 170 & 0 & +-- & $\mathrm{X}$ \\
\hline & & & 159 & 0 & --- & XI \\
\hline & & & 418 & -10 & --+ & XII \\
\hline & & & 1038 & -204 & -+- & XIII \\
\hline & & & 988 & -31 & -++ & XIV \\
\hline \multirow{4}{*}{$\sigma \sigma$} & \multirow{4}{*}{118} & \multirow{4}{*}{-2227} & $\overline{4741}$ & -4688 & --- & $\mathrm{XV}$ \\
\hline & & & 3687 & -2875 & -+- & XVI \\
\hline & & & 3626 & -3456 & +-- & XVII \\
\hline & & & 3533 & -579 & ++- & XVIII \\
\hline
\end{tabular}

with $f_{0}(500)$ at lower energy (poles I, II, and IV) while the second one splits into four states related with $f_{0}(1400)$ at higher energy (poles VI to IX). The pole in the $K \bar{K}$ channel at $E=(881-i 498) \mathrm{MeV}$, lying far from the physical axis in the uncoupled case, moves to the $K \bar{K}$ quasibound state at $E=(988-i 31) \mathrm{MeV}$ on sheet $-++\left(\right.$ pole XIV). This is the $f_{0}(980)$ resonance which lies quite close to the physical axis and therefore it strongly influences behaviour of the $\pi \pi$ scattering phase shifts near the $K \bar{K}$ threshold. The pole trajectory linking the corresponding poles in the uncoupled and coupled cases is drawn as a solid line in Fig. 4a. In this figure we have also indicated at several intermediate positions the percentage strength of the reduced interchannel couplings. The same broad $K \bar{K}$ pole can also move to the pole XIII at $E=(1038-i 204) \mathrm{MeV}$ on sheet -+- . Its trajectory is drawn as a dotted line in Fig. 4a. Poles III, V, X and XI, lying on the real axis of energy 
below the $\pi \pi$ threshold, appear when the interchannel coupling strengths are large enough. Their influence on the $\pi \pi$ phase shifts is, however, small. The extremely wide pole in the third channel at $E=(118-i 2227) \mathrm{MeV}$ goes to very distant states at energies above $3000 \mathrm{MeV}$. Therefore these states cannot be directly visible in the energy dependence of phase shifts or inelasticities in the three open channels. Large values of interchannel coupling constants (Table 1 in [7]) are responsible for large pole shifts as seen in Table 3 and Fig. 4a. This applies also to other solutions considered below (see Tables 1, 团 to 6 and Fig. 4b).

\section{3b) Solution $B$}

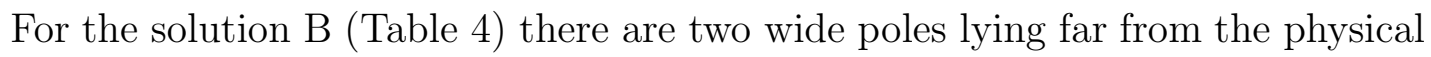
axis in the uncoupled $\pi \pi$ channel. The first pole at $(733-i 583) \mathrm{MeV}$ splits into

Table 4: Positions of $S$-matrix poles for the solution B (in $\mathrm{MeV}$ )

\begin{tabular}{|c|c|c|c|c|c|c|}
\hline \multirow[t]{2}{*}{ channel } & \multicolumn{2}{|c|}{ no couplings } & \multicolumn{2}{|c|}{ with couplings } & \multirow{2}{*}{$\begin{array}{c}\text { Sign of } \\
\operatorname{Im} k_{\pi} \operatorname{Im} k_{K} \operatorname{Im} k_{\sigma}\end{array}$} & \multirow[b]{2}{*}{ No } \\
\hline & ReE & $\operatorname{ImE}$ & $\operatorname{ReE}$ & $\operatorname{ImE}$ & & \\
\hline \multirow{4}{*}{$\pi \pi$} & \multirow{4}{*}{733} & \multirow{4}{*}{-583} & 332 & -114 & --- & I \\
\hline & & & 511 & -266 & -++ & II \\
\hline & & & 512 & -266 & -+- & III \\
\hline & & & 332 & -115 & --+ & IV \\
\hline \multirow{4}{*}{$\pi \pi$} & \multirow{4}{*}{999} & \multirow{4}{*}{-323} & 900 & -13 & --- & $\mathrm{V}$ \\
\hline & & & 1441 & -125 & -++ & VI \\
\hline & & & 1430 & -149 & -+- & VII \\
\hline & & & 942 & -25 & --+ & VIII \\
\hline \multirow{4}{*}{$K \bar{K}$} & \multirow{4}{*}{2744} & \multirow{4}{*}{-1698} & 3670 & -2263 & --- & IX \\
\hline & & & 3664 & -2240 & --+ & $\mathrm{X}$ \\
\hline & & & 3102 & -940 & +-- & XI \\
\hline & & & 3104 & -904 & +-+ & XII \\
\hline \multirow[b]{2}{*}{$\sigma \sigma$} & \multirow[b]{2}{*}{1284} & \multirow[b]{2}{*}{0} & 992 & -34 & -++ & XIII \\
\hline & & & 1421 & -54 & --+ & XIV \\
\hline \multirow[b]{2}{*}{$\sigma \sigma$} & \multirow[b]{2}{*}{1236} & \multirow[b]{2}{*}{0} & 956 & -36 & -+- & $\mathrm{XV}$ \\
\hline & & & 1411 & -85 & --- & XVI \\
\hline
\end{tabular}

four states I to IV. Pole II on sheet -++ and the corresponding zero lie close to the physical axis. Such a pole has an important influence on the $\pi \pi$ scattering amplitude below $1 \mathrm{GeV}$ and can be related to the $f_{0}(500)$ resonance. The second pole evolves from $(999-i 323) \mathrm{MeV}$ into a pair of poles at $(1441-i 125)$ and $(1430-i 149)$ $\mathrm{MeV}$ both related to the $f_{0}(1400)$ resonance. The two other shifted poles V and VIII remain close to $900 \mathrm{MeV}$. A very broad pole at $(2744-i 1698) \mathrm{MeV}$ in the uncoupled $K \bar{K}$ channel leads to four poles IX to XII lying far away from the physical region. Their influence on the scattering amplitudes is negligible. In Fig. 4b four interesting trajectories in the $k_{3}$ complex plane are drawn. The $\sigma \sigma$ bound state at $1284 \mathrm{MeV}$ 

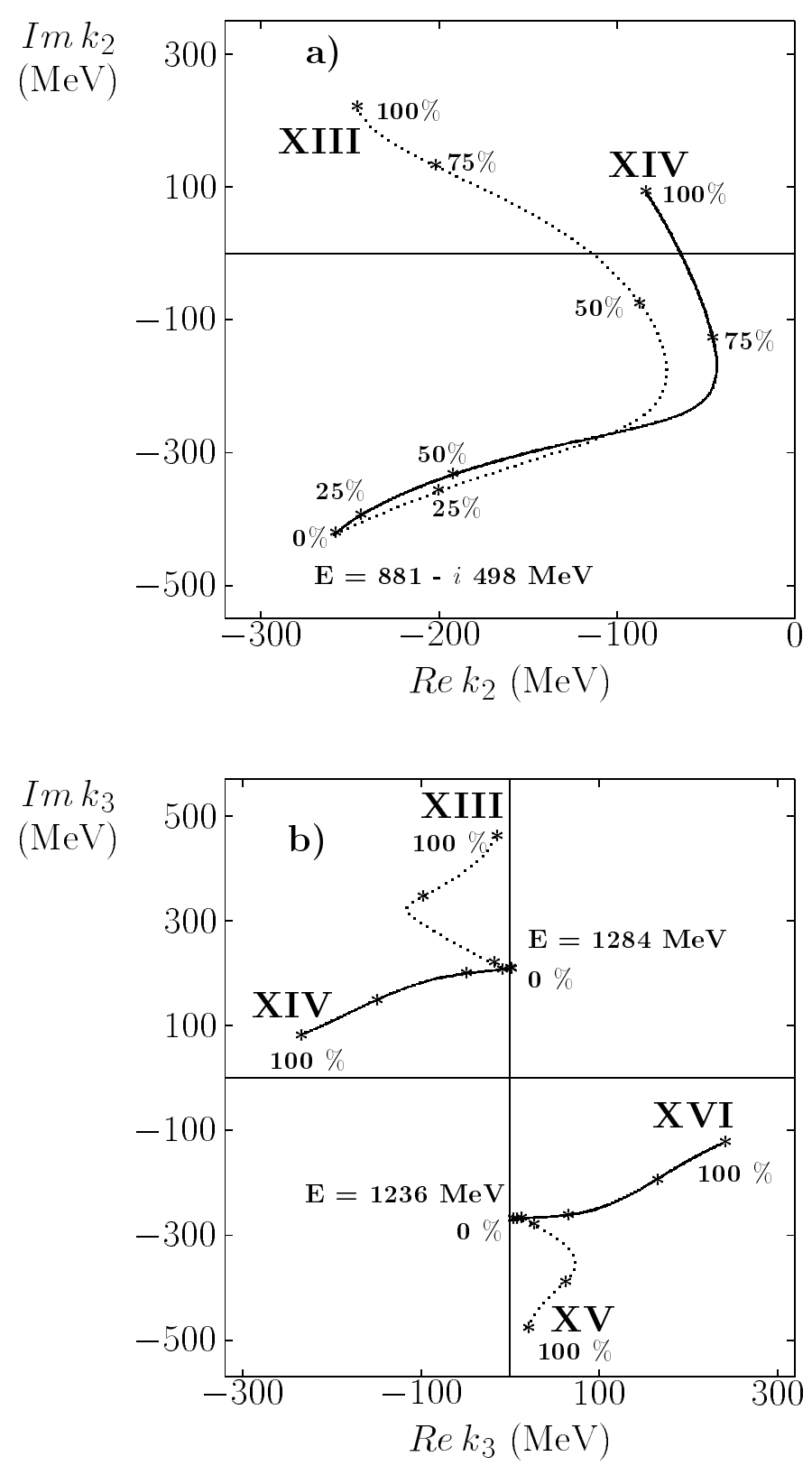

Figure 4: Pole trajectories in the complex momentum plane as a function of the percentage of the interchannel coupling strength: a) for the solution $\mathrm{A}$ in the $K \bar{K}$ channel, b) for the solution B in the $\sigma \sigma$ channel. Roman numbers and energies are taken from Table 3 for $\mathbf{a}$ ) and from Table 4 for $\mathbf{b}$ ). 
$\left(I m k_{3}>0\right)$ can either evolve to the $f_{0}(980)$ resonance (pole XIII) at $(992-i 34)$ $\mathrm{MeV}$ (dotted line) or to the $f_{0}(1400)$ (pole XIV) at (1421 - i54) MeV (solid line). Similarly the so-called quasibound state at $1236 \mathrm{MeV}\left(\operatorname{Im}_{3}<0\right)$ can either go to the pole XV at $(956-i 36) \mathrm{MeV}$ closely related to the $f_{0}(980)$ (dotted line) or to the $f_{0}(1400)$ resonance (pole XVI) at $(1411-i 85) \mathrm{MeV}$ (solid line).

\section{3c) Solution $E$}

In Table 1 the positions of poles for the solution $\mathrm{E}$ are shown. The first pole at $(542-i 307) \mathrm{MeV}$ evolves (as for the solution B) to four wide states. One of them (pole II) at $(533-i 254) \mathrm{MeV}$ on sheet -++ lies close to the physical region and can be related to $f_{0}(500)$. When the interchannel couplings are switched on, the second pole at $E=(1473-i 150) \mathrm{MeV}$, coming from the $\pi \pi$ channel in the uncoupled channel case, creates four poles V to VIII. The pole V on sheet - - and a zero related to the pole VI on sheet -++ are responsible for an increase of the $\pi \pi$ phase shifts around $1400 \mathrm{MeV}$ (see Fig. 2). Looking at inelasticities $\eta_{\pi}$ and $\eta_{K}$ in Fig. 1 one can see small bumps around $1400 \mathrm{MeV}$ caused by the pole V.

Table 5: Positions of $S$-matrix poles for the solution E (in $\mathrm{MeV}$ )

\begin{tabular}{|c|c|c|c|c|c|c|}
\hline \multirow[t]{2}{*}{ channel } & \multicolumn{2}{|c|}{ no couplings } & \multicolumn{2}{|c|}{ with couplings } & \multirow{2}{*}{$\begin{array}{c}\text { Sign of } \\
\operatorname{Im} k_{\pi} \operatorname{Im} k_{K} \operatorname{Im} k_{\sigma}\end{array}$} & \multirow[b]{2}{*}{ No } \\
\hline & $R e E$ & $\operatorname{ImE}$ & $R e E$ & $\operatorname{ImE}$ & & \\
\hline \multirow{4}{*}{$\pi \pi$} & \multirow{4}{*}{542} & \multirow{4}{*}{-307} & 600 & -355 & --- & $\mathrm{I}$ \\
\hline & & & 533 & -254 & -++ & II \\
\hline & & & 533 & -246 & -+- & III \\
\hline & & & 600 & -354 & --+ & IV \\
\hline \multirow{4}{*}{$\pi \pi$} & \multirow{4}{*}{1473} & \multirow{4}{*}{-150} & 1421 & -79 & --- & $\mathrm{V}$ \\
\hline & & & 1441 & -106 & -++ & VI \\
\hline & & & 1428 & -104 & -+- & VII \\
\hline & & & 1466 & -38 & --+ & VIII \\
\hline \multirow[b]{2}{*}{$K \bar{K}$} & \multirow[b]{2}{*}{441} & \multirow[b]{2}{*}{0} & 328 & -7 & --- & IX \\
\hline & & & 336 & -8 & --+ & $\mathrm{X}$ \\
\hline \multirow[b]{2}{*}{$K \bar{K}$} & \multirow[b]{2}{*}{990} & \multirow[b]{2}{*}{0} & 978 & -46 & -+- & XI \\
\hline & & & 990 & -34 & -++ & XII \\
\hline \multirow{4}{*}{$\sigma \sigma$} & \multirow{4}{*}{1565} & \multirow{4}{*}{-112} & 1703 & -271 & --- & $\overline{\mathrm{XIII}}$ \\
\hline & & & 1648 & -67 & -+- & XIV \\
\hline & & & 1673 & -77 & --+ & $\mathrm{XV}$ \\
\hline & & & 1624 & -175 & +-- & XVI \\
\hline
\end{tabular}

The $K \bar{K}$ antibound state at $441 \mathrm{MeV}$ transforms into two states IX and X lying far away from the physical axes in the $K \bar{K}$ and $\sigma \sigma$ complex momentum spaces. The $K \bar{K}$ bound state in the uncoupled case lies very close to the $K \bar{K}$ threshold at $E=990 \mathrm{MeV}$ and can evolve, when interchannel couplings are switched on, into two narrow states (poles XI and XII) on sheets -+- and -++ . Pole XII on sheet 
-++ lies closer than pole XI to the physical region and therefore can be related to $f_{0}(980)$. The last resonance at $(1565-i 112) \mathrm{MeV}$ evolves into four states at about $1700 \mathrm{MeV}$. As it can be seen in Fig. 2 the $\pi \pi$ phase shifts do not strongly increase around $1700 \mathrm{MeV}$. This fact is related to the positions of two singularities of the $S$-matrix having the strongest influence on the $\pi \pi$ scattering amplitude. One of them is the pole XIII on sheet - - - and the second is a zero also lying on sheet --- related to the pole XVI on sheet +-- . The zero and the pole lie on the same sheet, so they partially cancel each other and their influence on the energy dependence of the $\pi \pi$ amplitude is unusually small. The zero lies closer to both real and imaginary energy axes than the pole. Therefore in Fig. 2 a flat energy dependence of the $\pi \pi$ phase shifts between 1600 and $1750 \mathrm{MeV}$ is seen as well as a smooth increase above $1750 \mathrm{MeV}$ where the influence of the pole becomes larger than the action of the zero.

Another particular case of influence of $S$-matrix singularities on scattering amplitudes can be seen in Fig. 3b. A strong decrease of the $\sigma \sigma$ phase shifts for solution E, very well visible near threshold at $1350 \mathrm{MeV}$, can be understood if we take into account all the singularities of the $S_{33}$ element of the $S$-matrix. For this solution the value of parameter $\beta_{3}$ is very small $\left(\beta_{3}=92.7 \mathrm{MeV}\right)$ so the double zero of $S_{33}$ at $k_{3}=-i \beta_{3}$ is the closest singularity to the $\sigma \sigma$ threshold. This zero of $S_{33}$ is a result of the Jost function double pole at $k_{3}=-i \beta_{3}$ as it can be seen in the analytical expression for $J_{33}$ given in Eq. (A9) of [9]. One can calculate the $\sigma \sigma$ phase shifts keeping only this pole contribution to $J_{33}$. The result is drawn in Fig. $3 \mathrm{~b}$ as the dotted line denoted by " $\beta$ ". Comparison with the full calculation shows the dominance of this singularity over a large energy range. The poles related to the $f_{0}(1400)$ and $f_{0}(1710)$ only slightly disturb the energy dependence of the $\sigma \sigma$ phase shifts at about 1425 and $1625 \mathrm{MeV}$. This example shows that one cannot extract a complete information about resonances from the energy dependence of phase shifts. One furthermore needs to know the analytical structure of the $S$-matrix singularities.

Let us here note that in the Particle Data Table []] there is a state called $f_{J}(1710)$ in a mass range similar to that of our $f_{0}(1710)$, with a $J=0$ possibility not excluded. It has been recently shown that this $J=0$ assignment is more favorable than the $J=2$ one 18 .

\section{3d) Solution $\mathrm{F}$}

In the solution $\mathrm{F}$ there are both $K \bar{K}$ and $\sigma \sigma$ bound states in the uncoupled channel case (see Tables 2 and (6). In the fully coupled case, the $\sigma \sigma$ quasibound state with a width about $0.5 \mathrm{MeV}$ generates a very narrow $180^{\circ}$ jump of the $\pi \pi$ phase shifts at about $1350 \mathrm{MeV}$. The appearance of such a not well confirmed resonance cannot be excluded since the precision of the existing data is rather limited and the

$\chi^{2}$ values for the solution $\mathrm{F}$ are still acceptable. Apart from this narrow state, the solution $\mathrm{F}$ is similar to other solutions so in the further analysis we shall not study in detail properties of this solution.

Comparing different solutions we have seen that similar relatively narrow re- 
Table 6: Positions of $S$-matrix poles for the solution F (in MeV)

\begin{tabular}{|c|c|c|c|c|c|c|}
\hline \multirow[t]{2}{*}{ channel } & \multicolumn{2}{|c|}{ no couplings } & \multicolumn{2}{|c|}{ with couplings } & \multirow{2}{*}{$\begin{array}{c}\text { Sign of } \\
\operatorname{Im} k_{\pi} \operatorname{Im} k_{K} \operatorname{Im} k_{\sigma}\end{array}$} & \multirow[b]{2}{*}{ No } \\
\hline & ReE & $\operatorname{ImE}$ & ReE & $\operatorname{ImE}$ & & \\
\hline \multirow{4}{*}{$\pi \pi$} & \multirow{4}{*}{554} & \multirow{4}{*}{-377} & 691 & -511 & --- & $\mathrm{I}$ \\
\hline & & & 528 & -255 & -++ & II \\
\hline & & & 673 & -410 & -+- & III \\
\hline & & & 658 & -379 & --+ & IV \\
\hline \multirow{4}{*}{$\pi \pi$} & \multirow{4}{*}{1407} & \multirow{4}{*}{-80} & 1387 & -81 & --- & $\mathrm{V}$ \\
\hline & & & 1428 & -93 & -++ & VI \\
\hline & & & 1367 & -79 & -+- & VII \\
\hline & & & 1447 & -77 & --+ & VIII \\
\hline \multirow[b]{2}{*}{$K \bar{K}$} & \multirow[b]{2}{*}{497} & \multirow[b]{2}{*}{0} & 486 & -5 & --- & IX \\
\hline & & & 396 & -25 & --+ & $\mathrm{X}$ \\
\hline \multirow[b]{2}{*}{$K \bar{K}$} & \multirow[b]{2}{*}{968} & \multirow[b]{2}{*}{0} & 967 & -31 & -+- & $\mathrm{XI}$ \\
\hline & & & 993 & -42 & -++ & XII \\
\hline \multirow[b]{2}{*}{$\sigma \sigma$} & \multirow[b]{2}{*}{341} & \multirow[b]{2}{*}{0} & 250 & 0 & --- & XIII \\
\hline & & & 270 & 0 & -+- & XIV \\
\hline \multirow[b]{2}{*}{$\sigma \sigma$} & \multirow[b]{2}{*}{1353} & \multirow[b]{2}{*}{0} & 1349 & -0.2 & --+ & $\mathrm{XV}$ \\
\hline & & & 1349 & -0.3 & -++ & XVI \\
\hline
\end{tabular}

sonances can emerge from very different poles in the uncoupled channel cases depending on the set of the interaction parameters. Therefore one would need more data of a better precision to disentangle between phenomenologically good solutions $\mathrm{A}, \mathrm{B}$ and $\mathrm{E}$. In the next section we shall further discuss other properties of the meson-meson scattering amplitudes.

\section{Influence of $S$-matrix poles and zeroes on phase shifts and inelasticities near $f_{0}(1400)$ resonance}

Properties of the scattering and production amplitudes in the energy region near $1400 \mathrm{MeV}$ can be understood provided that we know the positions of the $S$-matrix singularities, especially the poles and zeroes close to the physical region. In the previous chapter we have seen that the $S$-matrix has many poles lying on different sheets. Now we would like to choose the most important poles and zeroes which influence the phase shifts and inelasticities and determine the resonance parameters.

The $S$-matrix elements $S_{i j}(i, j=1,2,3)$ can be written in terms of the Jost function of different arguments, for example

$$
S_{11}=\frac{D\left(-k_{1}, k_{2}, k_{3}\right)}{D\left(k_{1}, k_{2}, k_{3}\right)}
$$


Expressions for some other matrix elements can be found in [19]. All the $S$-matrix elements are inversely proportional to the Jost function $D\left(k_{1}, k_{2}, k_{3}\right)$ which has a zero on sheet - - at the channel momenta $k_{i d}(i=1,2,3)$ :

$$
D\left(k_{1 d}, k_{2 d}, k_{3 d}\right)=0
$$

The corresponding energy $E_{d}$ in the complex plane is given by

$$
E_{d}=2 \sqrt{k_{1 d}^{2}+m_{1}^{2}}=2 \sqrt{k_{2 d}^{2}+m_{2}^{2}}=2 \sqrt{k_{3 d}^{2}+m_{3}^{2}}
$$

where $m_{i}$ denote meson masses. Energies $E_{d}$ on sheet --- corresponding to the $f_{0}(1400)$ resonance are collected in Table 7 for our solutions $\mathrm{A}, \mathrm{B}, \mathrm{E}$ and $\mathrm{F}$. The knowledge of this pole position of the $S$-matrix is, however, not sufficient to describe the pion-pion phase shifts and inelasticities even at the energy closest to the pole. We should also know the zero of the numerator $D\left(-k_{1}, k_{2}, k_{3}\right)$ on sheet +++ close to the physical axis. This zero is in turn related to the zero of the denominator $D\left(k_{1}, k_{2}, k_{3}\right)$ on sheet -++ . The corresponding energies on sheet -++ are also given in Table 7. We can notice that both real and imaginary parts of energy are shifted on sheet -++ if we compare them with the corresponding parts of energy on sheet --- . The values of phase shifts and inelasticities depend on the zero of the numerator $D\left(-k_{1}, k_{2}, k_{3}\right)$ and on the zero of the denominator $D\left(k_{1}, k_{2}, k_{3}\right)$. This means that by measurements of the $\pi \pi$ phase shifts and inelasticities we cannot uniquely determine one single value of the resonance energy and one value of the resonance width related to the imaginary part of energy. Strong interchannel couplings are responsible for the energy shifts of zeroes found on different sheets.

Table 7: Energy positions of $S$-matrix poles related to $f_{0}(1400)$

\begin{tabular}{|c|c|c|c|c|}
\hline \multirow{2}{*}{ Solution } & \multicolumn{4}{|c|}{ Energy $($ in MeV) } \\
\cline { 2 - 5 } & sheet & sheet & sheet & sheet \\
& --- & -++ & -+- & --+ \\
\hline A & $1405-i 74$ & $1445-i 116$ & $1424-i 94$ & $1456-i 47$ \\
B & $1411-i 85$ & $1441-i 125$ & $1430-i 149$ & $1421-i 54$ \\
E & $1421-i 79$ & $1441-i 106$ & $1428-i 104$ & $1466-i 38$ \\
F & $1387-i 81$ & $1428-i 93$ & $1367-i 79$ & $1447-i 77$ \\
\hline
\end{tabular}

In vicinity of the $f_{0}(1400)$ resonance one can, however, built up an approximation to the $S$-matrix elements in three channels. This will furthermore allow us to understand the role played by the four poles whose energy positions in different sheets are given in Table 7 for our four solutions A, B, E and F.

We make an expansion of the Jost function $D\left(k_{1}, k_{2}, k_{3}\right)$ near its zero on sheet -- at $\left(k_{1 d}, k_{2 d}, k_{3 d}\right)$

$$
D\left(k_{1}, k_{2}, k_{3}\right) \approx\left(k_{1}-k_{1 d}\right) d_{1}
$$


Let us denote by $k_{i n}(i=1,2,3)$ the zero position of the same Jost function on sheet -++ . Then in the first approximation

$$
D\left(-k_{1}, k_{2}, k_{3}\right) \approx\left(k_{1}-k_{1 n}^{*}\right) c_{1} .
$$

Here $d_{1}$ and $c_{1}$ are complex constants. The quantity $1 / d_{1}$ is the residuum of the $1 / D\left(k_{1}, k_{2}, k_{3}\right)$ pole on sheet --- and $1 / c_{1}$ is the residuum of the pole on sheet -++ . Here we have used the property of the Jost function expressed by (11). The twin zero can sometimes be closer than the pole on sheet - - - to the physical region and therefore it can strongly influence the $S$-matrix element. The $\pi \pi S$ matrix element can be then approximated by

$$
S_{11}=\frac{k_{1}+p_{1}}{k_{1}-k_{1 d}} f_{1}
$$

where $f_{1}=c_{1} / d_{1}$ and $p_{1}=-k_{1 n}^{*}$. Similarly the $K \bar{K}$ and $\sigma \sigma S$-matrix elements can be written as

$$
\begin{aligned}
& S_{22}=\frac{D\left(k_{1},-k_{2}, k_{3}\right)}{D\left(k_{1}, k_{2}, k_{3}\right)} \approx \frac{k_{2}+p_{2}}{k_{2}-k_{2 d}} f_{2}, \\
& S_{33}=\frac{D\left(k_{1}, k_{2},-k_{3}\right)}{D\left(k_{1}, k_{2}, k_{3}\right)} \approx \frac{k_{3}+p_{3}}{k_{3}-k_{3 d}} f_{3},
\end{aligned}
$$

where $f_{2}$ and $f_{3}$ are complex constants, $p_{2}$ is the kaon complex momentum on sheet -+- and $p_{3}$ is the $\sigma$ momentum on sheet --+ for which the Jost functions in numerators vanish.

We have numerically checked, by comparison with the exact values, that the phase shifts and inelasticities for the solutions $\mathrm{A}, \mathrm{B}$ and $\mathrm{E}$, in the region of the $f_{0}(1400)$ resonance (effective mass range between 1350 and $1500 \mathrm{MeV}$ ), are qualitatively well described by the formulae (7) to (9). For the solution B the agreement is even quantitative since the percentage error is only of the order of $10 \%$ or less. This error is obtained if the coupling constants $f_{i}$ are calculated using the first order derivatives of the Jost function. The errors can be reduced further if we modify the complex factors $f_{1}, f_{2}$ and $f_{3}$ taking into account the second derivatives of the Jost function as explained in Appendix B. The solution E is somewhat particular since the region of $f_{0}(1400)$ is also influenced by a wide resonance $f_{0}(1710)$ (see Table 5). The phase shifts in the $\sigma \sigma$ channel are additionally affected by the second order zeroes at $k_{3}= \pm i \beta_{3}$ since the parameter $\beta_{3}$ is small in that case as already discussed in Section 3c.

\section{Limited applicability of the Breit-Wigner ap- proach}

We should note here that zeroes of the Jost function $D\left(k_{1}, k_{2}, k_{3}\right)$ on sheets ,,----++-+- and --+ are in general different, so the following inequalities 
between the corresponding complex momenta hold: $p_{1} \neq k_{1 d}, p_{2} \neq k_{2 d}$ and $p_{3} \neq k_{3 d}$. In particular, looking at the Table 7 we can notice that the poles on sheet --+ are shifted towards higher energy in comparison with poles on sheet --- . Also the corresponding width related to the imaginary part of energy is considerably reduced on sheet --+ for the solutions $\mathrm{A}, \mathrm{B}$ and $\mathrm{E}$. This fact has important consequences on the energy dependence of phase shifts and inelasticity parameters which will be different from those obtained using the Breit-Wigner form.

Let us now compare the approximations to the diagonal $S$-matrix elements (7) to (9) and the Breit-Wigner multichannel formula for the transition matrix elements

$$
T_{i j}^{B W}=\frac{1}{\sqrt{k_{i} k_{j}}} \frac{M \Gamma}{M^{2}-s-i M \Gamma} c_{i} c_{j} .
$$

In this equation $M$ is the mass and $\Gamma$ the width of a resonance, $c_{i}, c_{j}$ are real channel branching ratios and $c_{i}^{2}=\Gamma_{i} / \Gamma, \Gamma_{i}$ being the partial decay width. These formulae are valid if the resonance pole dominates the transition amplitude in the physical region and if background can be neglected. The corresponding $S$-matrix diagonal elements expressed by

$$
S_{i i}^{B W}=1+2 i k_{i} T_{i i}^{B W}
$$

are then written as

$$
S_{i i}^{B W}=\frac{s-\left[M^{2}+i M\left(2 \Gamma_{i}-\Gamma\right)\right]}{s-\left(M^{2}-i M \Gamma\right)} .
$$

Remembering that $s=E^{2}=4\left(k_{i}^{2}+m_{i}^{2}\right)$ we can approximate (12) by

$$
S_{i i}^{B W} \approx \frac{k_{i N}}{k_{i D}} \frac{k_{i}-k_{i N}}{k_{i}-k_{i D}}
$$

where

$$
k_{i D}^{2}=\frac{M^{2}}{4}-m_{i}^{2}-i \frac{M \Gamma}{4},
$$

and

$$
k_{i N}^{2}=\frac{M^{2}}{4}-m_{i}^{2}+i \frac{M}{4}\left(2 \Gamma_{i}-\Gamma\right) .
$$

While formally (13) looks very similar to (7), (8) and (9), it is very different from them since $k_{i D}$ and $k_{i N}$ have to satisfy the inequalities: $\operatorname{Re}_{i N} \leq \operatorname{Re}_{i D},\left|I m k_{i N}\right| \leq$ $\left|I m k_{i D}\right|$. These inequalities follow from an obvious inequality that each partial width $\Gamma_{i}$ is smaller than the total width $\Gamma$. In fact each Breit-Wigner $S_{i i}$ matrix element depends only on three real parameters $M, \Gamma$ and $\Gamma_{i}$ while the matrix elements (7), (8) and (9) depend on three complex parameters: one giving the position of the zero in the denominator, the second giving the zero of the numerator and the third parameter being $f_{i}$. 
The Breit-Wigner formula (10) is very often used to analyze experimental data in order to obtain a mass and a width of different resonant states [1]. It is widely believed that it should provide the same resonance parameters independently on the reaction channel in which the resonant signal is detected. Our analysis, however, puts some limits on a practical applicability of the Breit-Wigner approach. The point is that in each reaction channel not only the $S$-matrix pole plays a significant role but also the accompanying zero. The pole is common for all the reaction channels but the zero is different in each channel and it cannot be located simply by giving one number corresponding to a branching ratio or a channel coupling constant. This point will be discussed in detail in the two next sections. Here we can make the following remark. If one applies the Breit-Wigner formula to the data analysis in a particular channel then one can obtains distorted resonance parameters which are to some extend "averaged" over the pole and accompanying zero parameters. This effect might explain a fact that some resonant parameters "measured" in one coupled channel can be different from those obtained in another channels if the phenomenological model applied in the data analyses is essentially restricted to the Breit-Wigner formula.

\section{Branching ratios}

Branching ratios are important parameters of hadronic resonances. Usually they are obtained from experimental data applying the multichannel Breit-Wigner formula (10) with some background parametrization in a limited range of effective masses. In our model we do not use any arbitrary background parametrization nor the Breit-Wigner parametrization. We fully exploit the knowledge of the analytical structure of the $S$-matrix. Some $S$-matrix poles close to the physical range can be related to the scalar meson resonances as already discussed in Sections 2 and 3. From the position of a given pole in the complex energy plane we can deduce the mass of the resonance and its total width. A determination of the partial decay width in presence of two and three open channels is, however, a more complicated issue which we are going to discuss in this section.

\section{6a) Definitions}

Let us recall that our model satisfies the unitarity condition for the $S$ matrix: $S^{+} S=1$ and that the diagonal matrix elements are parametrized as

$$
S_{j j}=\eta_{j} e^{2 i \delta j}, \quad j=1,2,3,
$$

where $\eta_{j}$ inelasticities and $\delta_{j}$ the channel phase shifts. The nondiagonal elements are related to the non-diagonal reaction $T$-matrix elements by

$$
T_{j l}=\frac{1}{2 i} \frac{1}{\sqrt{k_{j} k_{l}}} S_{j l}, \quad j, l=1,2,3, \quad j \neq l .
$$


Expressions for $S_{j l}$ can be found in [19]. The diagonal $T$-matrix elements read

$$
T_{j j}=\frac{1}{2 i k_{j}}\left(S_{j j}-1\right), \quad j=1,2,3 .
$$

$T$-matrix elements satisfy the unitarity equations. For example, in the first channel above the $\sigma \sigma$ threshold

$$
I m T_{11}=k_{1}\left|T_{11}\right|^{2}+k_{2}\left|T_{12}\right|^{2}+k_{3}\left|T_{13}\right|^{2} .
$$

The total cross section in the first channels reads:

$$
\sigma_{11}^{\text {tot }}=\frac{8 \pi}{k_{1}} \operatorname{Im} T_{11} .
$$

The elastic cross section is given by

$$
\sigma_{11}^{e l}=8 \pi\left|T_{11}\right|^{2}
$$

and the transition cross sections from the channel 1 to the channels $j=2$ or 3 are expressed by

$$
\sigma_{1 j}=\frac{8 \pi}{k_{1}} k_{j}\left|T_{1 j}\right|^{2} .
$$

These cross sections satisfy the equation:

$$
\sigma_{11}^{t o t}=\sigma_{11}^{e l}+\sigma_{12}+\sigma_{13} .
$$

In Fig. 5 all the $\pi \pi$ cross sections corresponding to the solution $\mathrm{B}$ are shown. At low energies we see a huge peak with a maximum near $600 \mathrm{MeV}$. It can be attributed to a very wide scalar $\sigma$ meson. Near $1 \mathrm{GeV}$ one notices a very deep and narrow minimum which is related to the $f_{0}(980)$ meson. The fact that this resonance is seen as a dip and not, as in the most cases as, a maximum of the total cross section, is due to the special value of the elastic $\pi \pi$ phase shift which goes through $180^{\circ}$ slightly below $1 \mathrm{GeV}$. If it happens below the $K \bar{K}$ threshold the total cross section is equal to 0 . The next minimum of the total cross section is near $1500 \mathrm{MeV}$ where another resonance $f_{0}(1500)$ appears. Up to the $K \bar{K}$ threshold the $\pi \pi$ scattering is elastic but above the $K \bar{K}$ threshold the $\pi \pi$ to $K \bar{K}$ transition cross section becomes visible. The transition to the $\sigma \sigma$ channel is clearly visible above the third threshold at about $1350 \mathrm{MeV}$.

Numbers of meson pairs produced in each channel are proportional to the cross sections defined above so for example in the $\pi \pi$ channel we can define three branching ratios

$$
b_{1 j}=\frac{\sigma_{1 j}}{\sigma_{11}^{\text {tot }}}, \quad j=1,2,3 .
$$

These branching ratios obviously satisfy

$$
b_{11}+b_{12}+b_{13}=1 .
$$




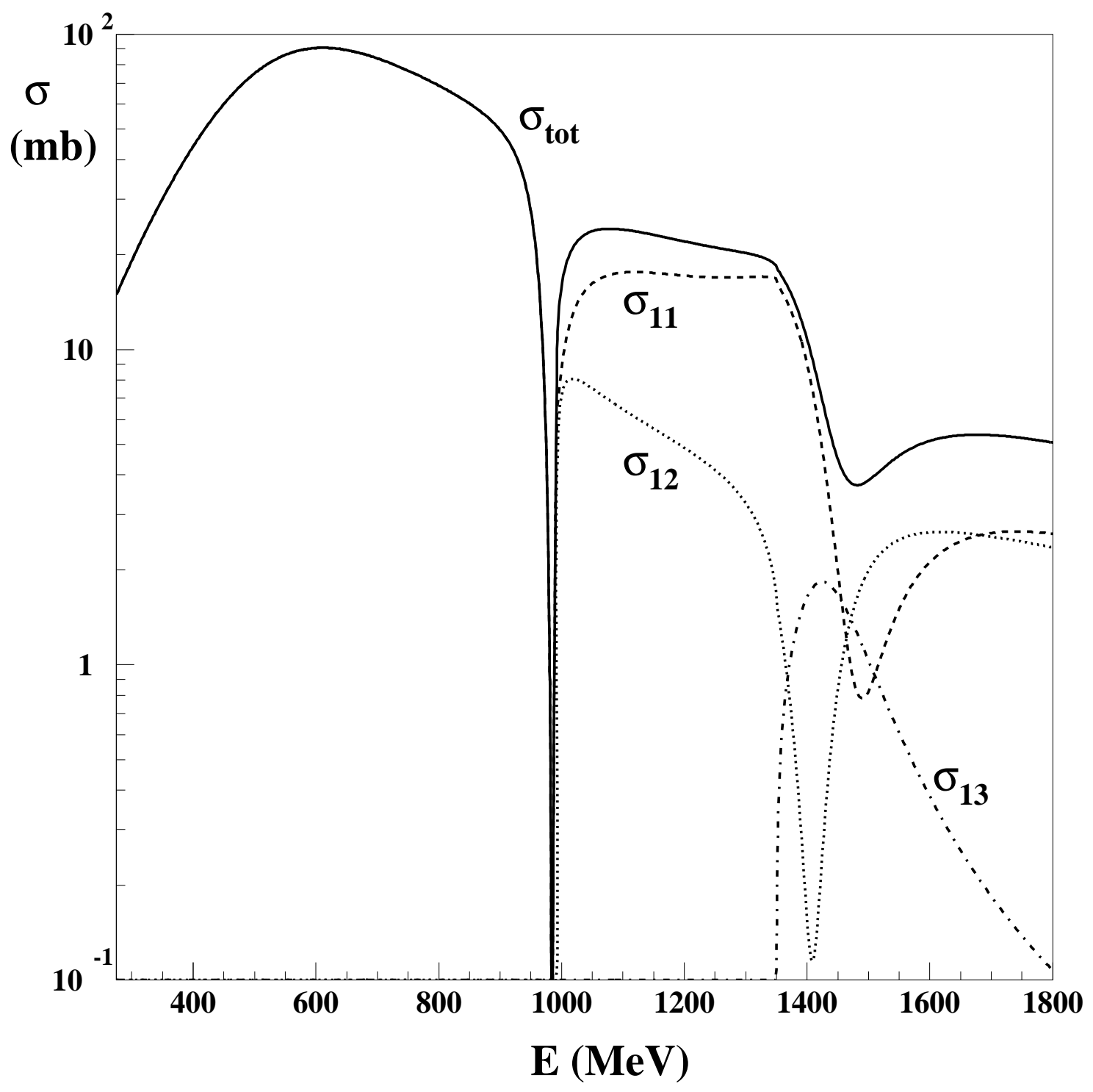

Figure 5: Energy dependence of cross sections $\sigma_{i j}$ for the solution B 
Cross sections similar to $\sigma_{1 j}$ and the corresponding branching ratios can be defined in channels 2 and 3 by changing index 1 into 2 and 3, respectively. In this way one can obtain nine branching ratios $b_{i j}(i, j=1,2,3)$. All these quantities as well as the cross sections are functions of energy or the effective mass. Below the third threshold where energy is smaller than $2 m_{3}$ but greater than $2 m_{2}$ the branching ratio matrix $b_{i j}$ reduces to $2 \times 2$ matrix containing only four nonzero matrix elements $b_{11}, b_{12}, b_{21}$ and $b_{22}$.

\section{6b) Discussion}

We shall discuss in detail the behaviour of branching ratios for the solution B, plotted in Fig. 6. Energy dependence of branching ratios for solutions $\mathrm{A}$ and $\mathrm{E}$ is similar to that for solution $\mathrm{B}$. We do not expect that the energy behaviour of the branching ratios for the "up-flat" solutions (solutions C and D in [7]) will be qualitatively different from that of the "down-flat" solutions A and B.

At first let us discuss the behaviour of the "elastic" branching ratio $b_{11}$ as a function of energy in the pion-pion channel (see Fig. 6a). At the $K \bar{K}$ threshold $b_{11}=1$ but it decreases enormously steeply with increasing energy to about 0.6 (solution A and E), 0.55 (solution B) or 0.5 (2-channel "down-flat" solution of [7]) already at the energy close to $1 \mathrm{GeV}$. This steep decrease is related to the opening of the $K \bar{K}$ channel. Next $b_{11}$ steadily increases to a maximum close to 0.9 for the above solutions at the energy reaching about $1.4 \mathrm{GeV}$. At this energy the third threshold is open for the solutions $\mathrm{A}, \mathrm{B}$ and $\mathrm{E}$. For $E>1.4 \mathrm{GeV} b_{11}$ decreases very fast to a minimum close to 0.2 for three cases A, B and E and to 0 for 2-channel "down-flat" solution. Then it rises again especially steeply for the latter solution and slower for the solutions A and B. This minimum is closely related to the presence of $f_{0}(1400)$.

The $b_{12}$ branching ratio is equivalent to the transition probability of two pions into a pair of two kaons. Below the third threshold $\left(E<2 m_{3}\right) b_{12}=1-b_{11}$, so its behaviour is completely determined by $b_{11}$. The $b_{12}$ rises very steeply from the $K \bar{K}$ threshold attaining a maximum near $1 \mathrm{GeV}$, then decreases to a minimum value at about $1.4 \mathrm{GeV}$ (see Fig. 6a). At this energy, however, the third threshold opens and the transition to the $\sigma \sigma$ channel becomes possible as stated by (25). An effect of the interplay between three channels is that $b_{12}$ rises above $1.4 \mathrm{GeV}$ attaining a maximum and then decreases rather slowly with energy for $E>1.5 \mathrm{GeV}$.

Above the third threshold in the $\pi \pi$ and $K \bar{K}$ channels a nonzero fraction of the channel total cross section originates from the transition to the $\sigma \sigma$ state. In the $\pi \pi$ channel the branching ratio $b_{13}$ forms a regular maximum at $E=1.48 \mathrm{GeV}, 1.46$ $\mathrm{GeV}$ and $1.48 \mathrm{GeV}$ for the solutions $\mathrm{A}, \mathrm{B}$ and $\mathrm{E}$, respectively. The width of this peak is about 110 to $120 \mathrm{MeV}$ (see Fig. 6a). The position and the width of this peak corresponds very closely to the parameters determined for the total width $f_{0}(1500)$ by the Crystal Barrel Collaboration in different channels [1].

In the second channel the $b_{21}$ element describes the ratio of the $K \bar{K}$ to $\pi \pi$ transition cross section to the total $K \bar{K}$ cross section. At the $K \bar{K}$ threshold the annihilation cross section $\sigma_{21}$ tends to infinity and the elastic cross section is finite, 

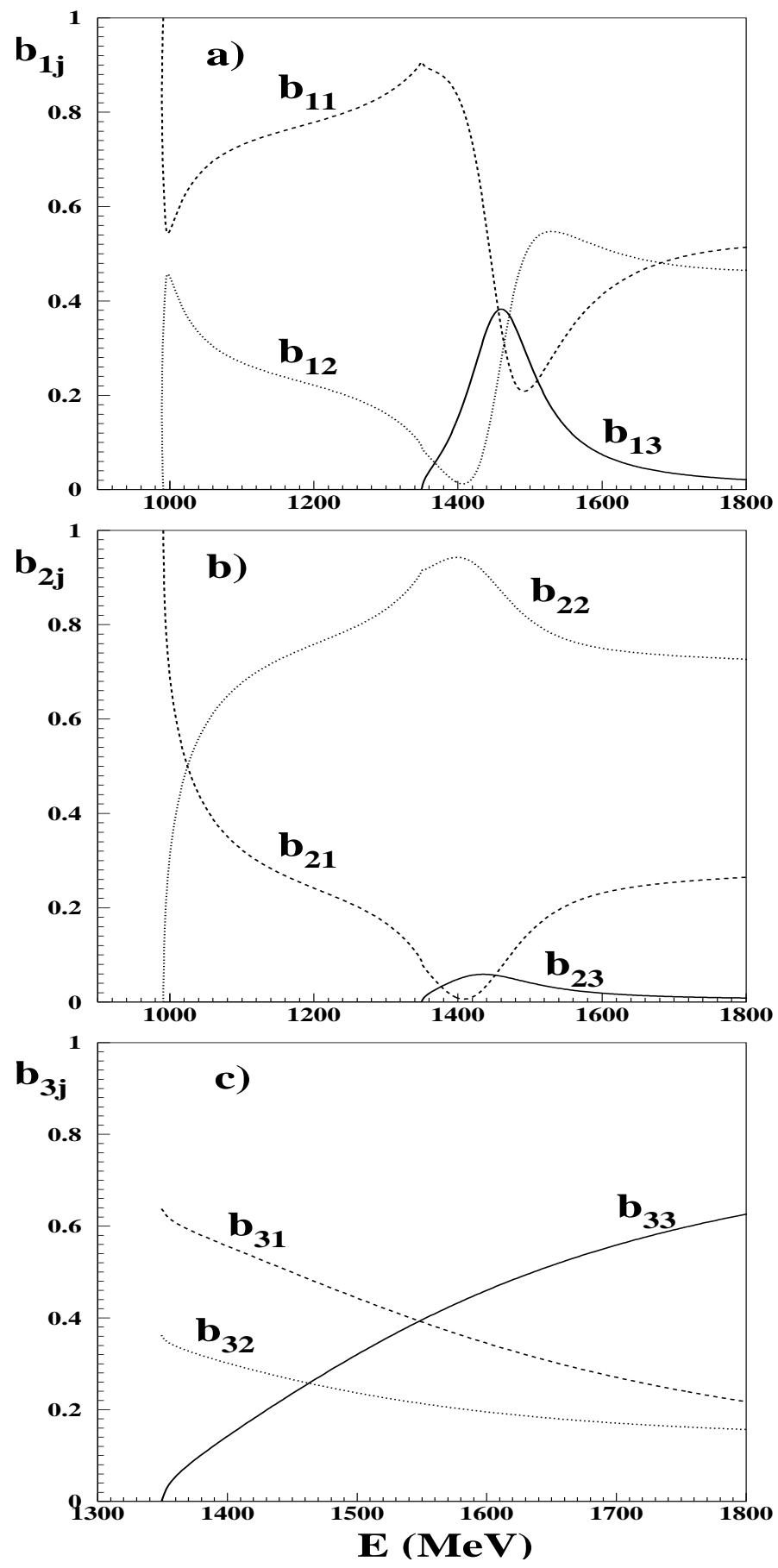

Figure 6: Energy dependence of branching ratios $b_{i j}$ for the solution B. Dashed line corresponds to $j=1$, dotted line to $j=2$ and solid line to $j=3$. In a) $i=1(\pi \pi$ channel $)$, in b) $i=2$ ( $K \bar{K}$ channel $)$ and in fig. c) $i=3$ ( $\sigma \sigma$ channel $)$. 
therefore the $b_{21}$ coefficient is equal to 1 . With increasing energy it decreases to a quite deep minimum close to 0 at about $1.4 \mathrm{GeV}$. At higher energy it rises slowly again for all three solutions. The transition element $b_{23}$ starts from 0 at the $\sigma \sigma$ threshold, reaches rather quickly a maximum and then drops smoothly (see Fig. $6 \mathrm{~b})$.

Two branching ratios in the third channel, namely $b_{31}$ and $b_{32}$, decrease from the large values at the $\sigma \sigma$ threshold. Their behaviour is characteristic for the annihilation reactions. On contrary, the elastic branching ratio $b_{33}$ rises monotonically with energy as shown in Fig. 6c.

6c) Average branching ratios near the $f_{0}(980)$ resonance

The $f_{0}(980)$ resonance lies very close to the $K \bar{K}$ threshold and this fact has a very important consequence on the experimental determination of the partial widths of this resonance for the decay into $\pi \pi$ and $K \bar{K}$ channels. The $\pi \pi$ channel is open below the $K \bar{K}$ threshold so for this channel we can determine the averaged $\pi \pi$ branching ratio $b_{11}$ choosing a range of the $\pi \pi$ effective mass ranges centered at the resonance mass $M_{s}$ with a maximum energy value equal to $M_{\max }$. This value should be larger than $M_{s}$ by more than the resonance width. This width is still not well defined experimentally. In [1] the full width is 40 to $100 \mathrm{MeV}$. In our three channel analyses [7] it was between 60 and $70 \mathrm{MeV}$ and larger than these numbers by 10 to $30 \mathrm{MeV}$ for two channel fits. Therefore we can choose the value $M_{\max }=1100 \mathrm{MeV}$ as the upper limit in the integration of the $\pi \pi$ or $K \bar{K}$ effective mass distributions. The average $\pi \pi$ branching ratio is thus defined as follows:

$$
<b_{11}>=\frac{1}{2\left(M_{\max }-M_{s}\right)} \int_{2 M_{s}-M_{\max }}^{M_{\max }} b_{11}(E) d E .
$$

The average $K \bar{K}$ branching ratio over the same energy interval is

$$
<b_{12}>=1-<b_{11}>\text {. }
$$

Since $b_{12}=0$ below the $K \bar{K}$ threshold

$$
<b_{12}>=\frac{1}{2\left(M_{\max }-M_{s}\right)} \int_{2 m_{2}}^{M_{\max }} b_{12}(E) d E .
$$

The mass values $M_{s}$ corresponding to the $f_{0}(980)$ resonance position on sheet -++ are 993, 989, 992 and $990 \mathrm{MeV}$ for two channel down-flat model and three channel models A, B and E, respectively [7]. After integration over energy we have obtained averaged $\left\langle b_{12}\right\rangle$ values of $0.191,0.156,0.170$ and 0.158 for the above four solutions corresponding to different sets of interaction parameters. Above numbers should not be, however, compared with the experimental value $21.9 \pm 2.4 \%$ quoted as $\Gamma(K \bar{K}) /[\Gamma(\pi \pi)+\Gamma(K \bar{K})]$ in the previous editions of the Reviev of Particle Properties (see for example 20]). The reason is that the numbers written under the title " $\Gamma_{\pi \pi} /\left(\Gamma_{\pi \pi}+\Gamma_{K K}\right)$ " in [20, 1] are the values of inelasticity coefficient $\eta_{a v}$, defined 
below, and therefore they are not equal to the partial branching ratios of $f_{0}(980)$. This difference is important since the branching ratios defined by (24) depend not only on the inelasticity coefficient $\eta$ but also on the $\pi \pi$ and $K \bar{K}$ phase shifts.

Experimentally the $\eta_{a v}$ value was obtained from the data on the $\pi^{-} p \longrightarrow K_{S}^{0} K_{S}^{0} n$ reaction using the relation:

$$
\eta^{2}(E)=1-\frac{\sigma\left(\pi^{+} \pi^{-} \longrightarrow f_{0}(980) \longrightarrow K_{S}^{0} K_{S}^{0}\right)}{\sigma_{u}},
$$

where $\sigma_{u}=\pi /\left(6 k^{2}\right)$ is the unitary limit of the $\pi^{+} \pi^{-} \rightarrow K_{S}^{0} K_{S}^{0}$ scalar isoscalar cross section with $k$ being the $K_{S}^{0}$ momentum in the $K_{S}^{0} K_{S}^{0}$ c.m. system. Let us remark that from the unitarity condition, below opening of the third channel, one has $\eta \equiv \eta_{1} \equiv \eta_{2}$. Thus one can define

$$
\eta_{a v}=\left(\frac{1}{E_{\max }-2 m_{K}} \int_{2 m_{K}}^{E_{\max }} d E \eta^{2}(E)\right)^{1 / 2}
$$

and use $E_{\max } \approx 1.1 \mathrm{GeV}$ as a good representation of the upper experimental limit of the $K_{S}^{0} K_{S}^{0}$ effective energy (see [21] for a discussion of experimental uncertainties). Experimental values of $\eta_{a v}$ obtained in [21, 22, 23] are equal to $0.67 \pm 0.09,0.78 \pm 0.03$ and $0.81_{-0.04}^{+0.09}$ respectively. Our calculations give lower values: $0.487,0.496$ and 0.494 for the solutions A, B and E, respectively. We have also calculated $\eta_{a v}$ values 0.494 and 0.761 corresponding to the two-channel "down-flat" solution of [7] and to the two-channel set 1 result of [9], respectively. Here we should stress that the last number is a result of the fit to the data for the reactions $\pi^{-} p \longrightarrow K^{-} K^{+} n$ and $\pi^{+} n \longrightarrow K^{-} K^{+} p$ of [15] while the four previous numbers around 0.49 are based on inelasticities measured in the $\pi^{-} p_{\uparrow} \rightarrow \pi^{+} \pi^{-} n$ [8]. The difference between above two-channel fits is therefore due to use of different experimental data sets. Problems with normalization of the $\pi \pi \rightarrow K \bar{K}$ cross sections have already been discussed by Morgan and Pennington in [24] (see in particular Fig. 4 therein) and Bugg, Sarantsev and Zou in [25]. The values of inelasticity $\eta$ from [8], which were later used in [7], correspond to the elastic $\pi \pi \rightarrow \pi \pi$ reaction. Although the errors are quite large the $\eta$ values found in [8] near the $f_{0}(980)$ resonance are visibly lower then those of [15]. This difference, however, could be partially explained by a possible contribution of channels other than $\pi \pi$ and $K \bar{K}$.

This difference between the data can also be seen in the calculation of $\left\langle b_{12}\right\rangle$. If we use the parameters corresponding to the set 1 of [9] then $\left\langle b_{12}\right\rangle=0.055$. This low value is related to rather low mass of $f_{0}(980)$ equal to $973 \mathrm{MeV}$ and to its width 29 $\mathrm{MeV}$ obtained in [9]. In the fits described in [7] we have obtained the $f_{0}(980)$ masses close to $990 \mathrm{MeV}$ and substantially larger values of the $f_{0}(980)$ width. Authors of [9] and [26] have also improperly used other definition of "branching ratio" in their Eq. (52) and in Eqs. (33) to (35), respectively. Those equations gave essentially the averaged values of $1-\eta^{2}$ which should not be directly compared to $\eta_{a v}$.

One remark is in order here. If all the transition amplitudes are dominated by a single Breit-Wigner resonance as in (10) then the branching ratio 2x2 matrix has 
to satisfy the following relations: $b_{21}=b_{11}$ and $b_{22}=b_{12}$. These relations are not satisfied above the $f_{0}(980)$ resonance energy (see Fig. 6). It means among others that the transition amplitudes cannot be simply described in terms of a single BreitWigner formula.

In Fig. 6a,b we see that the element $b_{21}$ is in general larger than $b_{12}$ especially near the $K \bar{K}$ threshold where $b_{21}$ tends to 1 while $b_{12}$ goes to zero. Nevertheless there is rather wide range of energies between 1.15 and $1.4 \mathrm{GeV}$ where both ratios are quite close each other.

\section{6d) Average branching ratios in the range 1100 - $1420 \mathrm{MeV}$}

In [27] the mass range 1100 to $1420 \mathrm{MeV}$ has been chosen to compare the ratio of $\pi \pi$ to $K \bar{K}$ pairs produced by incoming pions on a polarized target at about 18 $\mathrm{GeV} / \mathrm{c}$. The $K \bar{K}$ branching ratio was $6.4_{-2.0}^{+1.6} \%$.

We have calculated the average values of $b_{12}$ in this energy region obtaining 0.189 , 0.175, 0.166 and 0.167 for the two-channel "down-flat" and three-channel solutions A, B and E, respectively. These values are higher than the experimental result of [27]. One should, however, remember that the experimental errors of phase shifts and inelasticities are large (see [7]) so the theoretical errors of $b_{12}$ are also large. For completeness we give the value 0.099 corresponding to the data set 1 of [9]. In order to get this value and the other four values written above we used the ordinary averaging procedure

$$
\bar{b}_{12}=\frac{1}{M_{\max }-M_{\min }} \int_{M_{\min }}^{M_{\max }} b_{12}(E) d E
$$

and not the definition (28) which is applicable only near the $K \bar{K}$ threshold. Here one should once again remind the confusion concerning the definitions of the branching ratios as discussed in subsection $6 \mathrm{c}$, namely the value $B_{a v}=16 \pm 1 \%$ calculated in [9] should not have been compared with the experimental branching ratio $6.4_{-2.0}^{+1.6 \%}$.

\section{6e) Average branching ratios near the $f_{0}(1400)$ resonance}

In presence of three open channels the branching ratio matrix $\boldsymbol{b}$ has 9 elements. As already discussed in $6 \mathrm{~b}$, behaviour of these elements in three different channels $\pi \pi, K \bar{K}$ and $\sigma \sigma$ is shown in Fig. 6 for the solution B. For the solutions A and $\mathrm{E}$ the general shape of curves is quite similar although numerical values of various branching ratios differ. In [7] we have found that the width of the scalar resonance called here $f_{0}(1500)$ varies between 90 and $180 \mathrm{MeV}$ depending on the solution. Now we can choose the energy interval $1350 \mathrm{MeV}$ to $1500 \mathrm{MeV}$ to present the averaged values of the branching ratios in the form of $3 \times 3$ matrix. The elements of the last line of this matrix are averaged over the energies larger than the third threshold energy equal to $2 m_{3}$. For the solutions $\mathrm{A}, \mathrm{B}$ and $\mathrm{E}$ we obtain

$$
\overline{\boldsymbol{b}}_{A}=\left(\begin{array}{ccc}
0.636 & 0.127 & 0.237 \\
0.061 & 0.844 & 0.095 \\
0.300 & 0.407 & 0.293
\end{array}\right)
$$




$$
\begin{array}{rlrl} 
& & & \\
& & & \\
& & \left(\begin{array}{ccc}
0.608 & 0.163 & 0.229 \\
0.054 & 0.900 & 0.045 \\
0.529 & 0.286 & 0.185
\end{array}\right) \\
\text { and } & \overline{\boldsymbol{b}}_{E}= & \left(\begin{array}{lll}
0.604 & 0.133 & 0.263 \\
0.063 & 0.813 & 0.124 \\
0.120 & 0.156 & 0.724
\end{array}\right)
\end{array}
$$

In the $K \bar{K}$ channel $\bar{b}_{22}$ dominates over $\bar{b}_{21}$ and $\bar{b}_{23}$. Here the probabilities of the $K \bar{K}$ to $\pi \pi$ or $\sigma \sigma$ transitions are quite small. In the $\sigma \sigma$ channel, however, for the solutions $\mathrm{A}$ and $\mathrm{B}$ there are strong transitions from $\sigma \sigma$ to $\pi \pi$ or $K \bar{K}$ transitions which actually are comparable to the transitions from the $\pi \pi$ channel to $\sigma \sigma$ channels.

In [28] the branching ratios for the $f_{0}(1500)$ decay into five channels, $\pi \pi, \eta \eta$, $\eta \eta^{\prime}, K \overline{\bar{K}}$ and $4 \pi$, are given as $29,5,1,3$ and $62 \%$, respectively. The two main disintegration channels are $\pi \pi$ and $4 \pi$. In this model the $4 \pi$ channel is represented by the effective $\sigma \sigma$ channel and we also obtain large fractions for the averaged branching ratios $\bar{b}_{11}$ and $\bar{b}_{13}$. If we calculate the ratios $b_{13} / b_{11}$ exactly at $1500 \mathrm{MeV}$ then we obtain numbers 2.4, 1.2 and 2.3 for the solutions A, B and E, respectively. These numbers illustrate the importance of the $4 \pi$ channel in agreement with experimental result of [28]. In [29] the ratio $r=\left(B\left[f_{0} \rightarrow K \bar{K}\right] / B\left[f_{0} \rightarrow \pi \pi\right]\right) k_{1} / k_{2}=0.24 \pm 0.09$ is calculated (here by $f_{0}$ we mean $f_{0}(1500)$ ). If we define the ratio $\bar{b}_{12} / \bar{b}_{11}$ then we obtain values $0.20,0.27$ and 0.22 for the solutions $\mathrm{A}, \mathrm{B}$ and $\mathrm{E}$, respectively. These values are close to $r$. From the partial decay widths of the $f_{0}(1500)$ given in [25] one can calculate $\Gamma_{K \bar{K}} / \Gamma_{\pi \pi} \approx 0.10 \pm 0.05$ which is smaller than $r$ but still consistent within the experimental errors. We know, however, that extraction of the branching ratios from experiment is a difficult task as it is, for example discussed in [30]. We should mention, however, that the average branching ratios depend quite sensitively on the energy bin chosen in the actual calculation as it can be seen in Fig. 6a. Furthermore we see that the branching ratio $b_{12}$, corresponding to the $\pi \pi \rightarrow K \bar{K}$ transition, is very small around $1420 \mathrm{MeV}$, close to the position of our $f_{0}(1400)$ resonance poles. This is in qualitative agreement with the small number for the $K \bar{K}$ branching ratio $(3 \%)$ given in [28].

\section{Coupling Constants}

Coupling constants are useful physical quantities related to the residuum of the $S$-matrix at its complex pole value

$$
s_{R}=M^{2}-i M \Gamma,
$$

where $M$ is a resonance mass and $\Gamma$ its total width. We define the coupling constants $g_{i}$ by the formula

$$
\frac{g_{i} g_{j}}{4 \pi}=i \sqrt{s_{R}} \lim _{s \rightarrow s_{R}}\left[\left(s-s_{R}\right) \frac{S_{i j}(s)}{\sqrt{k_{i} k_{j}}}\right] .
$$


The product of the constants $c_{i} c_{j}$ appearing in the Breit-Wigner formula (10) is proportional to $g_{i} g_{j}$ :

$$
c_{i} c_{j}=\frac{\left[\left(s_{R} / 4-m_{i}^{2}\right)\left(s_{R} / 4-m_{j}^{2}\right)\right]^{1 / 4}}{2 M \Gamma \sqrt{s_{R}}} \frac{g_{i} g_{j}}{4 \pi} .
$$

In this way the diagonal Breit-Wigner coupling constant corresponding to the matrix element $S_{i i}$ given by (12) reads

$$
\frac{g_{i B W}^{2}}{4 \pi}=2 M \Gamma_{i} \frac{\sqrt{s_{R}}}{k_{i D}},
$$

where $k_{i D}$ is defined by (14). One can notice that the knowledge of $g_{i}^{2} / 4 \pi$ in the Breit-Wigner approach is equivalent to a determination of the partial width $\Gamma_{i}$. The two other independent quantities are $M$ and $\Gamma$.

The coupling constants corresponding to the approximations ([7 to 9) are:

$$
\frac{g_{i}^{2}}{4 \pi}=8 i \sqrt{s_{R}} f_{i}\left(k_{i d}+p_{i}\right)
$$

They are complex and depend on six parameters in contrast to (35) where only three parameters appear.

Using (33) we have calculated the values of the coupling constants for three solutions $\mathrm{A}, \mathrm{B}$ and $\mathrm{E}$ at two resonances $f_{0}(980)$ and $f_{0}(1400)$. They are shown in Tables 8 and 9, respectively. We can notice that the dispersion of the values $\left|g_{1}\right|^{2} / 4 \pi$ corresponding to the $\pi \pi$ channel is the smallest one since we have mostly fitted the $\pi \pi$ data. The worst situation is in the $\sigma \sigma$ channel where there are no data available. In Table 9 we can notice that the $K \bar{K}$ coupling constants at the $f_{0}(1400)$ resonance are much smaller than the $\pi \pi$ coupling constants contrary to the $f_{0}(980)$ resonance as seen in Table 8. The knowledge of the coupling constants is not sufficient to describe fully the $S$-matrix elements since the coupling constants are only related to the poles. We need also positions of the zeroes as discussed in Sect. 4.

Table 8: Coupling constants of $f_{0}(980)$ at the pole $-++\left(\right.$ in $\left.\mathrm{GeV}^{2}\right)$

\begin{tabular}{|c|c|c|}
\hline Solution & $\frac{\left|g_{1}\right|^{2}}{4 \pi}$ & $\frac{\left|g_{2}\right|^{2}}{4 \pi}$ \\
\hline A & 0.37 & 1.84 \\
B & 0.41 & 1.33 \\
E & 0.40 & 1.94 \\
\hline
\end{tabular}


Table 9: Coupling constants of $f_{0}(1400)$ at the pole $---\left(\right.$ in $\left.\mathrm{GeV}^{2}\right)$

\begin{tabular}{|c|c|c|c|}
\hline Solution & $\frac{\left|g_{1}\right|^{2}}{4 \pi}$ & $\frac{\left|g_{2}\right|^{2}}{4 \pi}$ & $\frac{\left|g_{3}\right|^{2}}{4 \pi}$ \\
\hline $\mathrm{A}$ & 0.580 & 0.106 & 0.091 \\
$\mathrm{~B}$ & 0.607 & 0.233 & 0.433 \\
$\mathrm{E}$ & 0.579 & 0.107 & 0.217 \\
\hline
\end{tabular}

\section{Phenomenological parametrizations of multichannel amplitudes}

Following our studies described in previous chapters we shall here make some proposal for a simple parametrization of multichannel amplitudes. This parametrization, based on existence of poles and zeroes of the $S$-matrix, has limited energy range of applicability. If there is some evidence of the presence of a well separated resonance in some energy region one can use expressions (7) to (9) to describe the diagonal $S$-matrix elements. The corresponding diagonal $T$-matrix elements (18) read:

$$
T_{i i}=\frac{1}{k_{i}}\left(f_{i}-1+f_{i} \frac{k_{i d}+p_{i}}{k_{i}-k_{i d}}\right) .
$$

From (16) one can obtain the phase shifts and inelasticities in each channel provided that the three complex parameters $\left(k_{i d}, p_{i}\right.$ and $\left.f_{i}\right)$, determined in a fit to some data, satisfy the unitarity conditions $\left|S_{i}\right|^{2} \leq 1$ in a given energy range. The off-diagonal $S$-matrix elements (17) can then be directly calculated as explained in [19] for the three channel case.

The fitted parameters $k_{i d}$, related in all channels by the energy conservation (4), provide us with the resonance energy position and its width. Other complex parameters $p_{i}$ are, however, not related by (俩) since they correspond to zeroes on different sheets. If we consider a three-channel fit and if the resonance appears well above the third threshold then the pole lies on sheet --- which means that $I m k_{i d}<0$. The effective parameters $f_{i}$ will take into account the second order derivative corrections to the approximated Jost function as explained in Appendix $\mathrm{B}$ (see in particular equations (B9) to (B12)).

In Sect. 5 we have discussed the Breit-Wigner approximation. Our formula (37) can be reduced to the Breit-Wigner one if $f_{i}=1$ and $R e k_{i d} \approx-R e p_{i}$. Dynamically $f_{i}=1$ means that the derivatives of the Jost functions in numerators and denominators of the $S_{i i}$ matrix elements (like in (Q)) are identically the same (see for example (B10)). The phenomenology related to the formula (37) is richer than that corresponding to the Breit-Wigner multichannel one (10) since it depends on six real parameters while the latter one depends only on three real parameters 
in each channel. If one adds the so-called elastic background to the Breit-Wigner amplitude $T_{i i}^{B W}$ then one gets the four-parameter formula

$$
T_{i i}=\frac{e^{2 i \delta_{B}}-1}{2 i k_{i}}+e^{2 i \delta_{B}} T_{i i}^{B W}
$$

where $\delta_{B}$ is a background phase. Even introducing an inelasticity $\eta_{B}$ to the background amplitude like

$$
T_{i i}=\frac{\eta_{B} e^{2 i \delta_{B}}-1}{2 i k_{i}}+\eta_{B} e^{2 i \delta_{B}} T_{i i}^{B W}
$$

leads to a five-parameter formula. It is only when one enlarges the number of independent parameters in the $T_{i j}^{B W}(10)$, by allowing $c_{i}$ to be complex, that (39) is essentially equivalent to our approximation (37). Limitations of the Breit-Wigner formula have been already pointed out in previous sections. Here we see that a knowledge of the $S$-matrix poles is not sufficient to construct the scattering amplitudes. We must also know positions of the nearby zeroes $S$-matrix in order to describe the data with sufficient accuracy.

A case in which one encounters more than a single resonance lying near the threshold should be treated differently especially if one wishes to study the energy range containing that threshold. Then in general more than one $S$-matrix pole should be taken into account. In our analysis near $1400 \mathrm{MeV}$ such a secondary pole appears on sheet --+ close to the physical region. Therefore this pole is included in Table 10 showing average values of masses and widths of scalar resonances $f_{0}(500)$, $f_{0}(980)$ and $f_{0}(1400)$ obtained by us. Its mass is higher and width is smaller than those of the --- pole.

Table 10: Average masses and widths of resonances $f_{0}(500), f_{0}(980)$ and $f_{0}(1400)$ found in our solutions A, B, E and F. Here errors represent the maximum departure from the average.

\begin{tabular}{|c|c|c|c|}
\hline resonance & mass $(\mathrm{MeV})$ & width $(\mathrm{MeV})$ & sheet \\
\hline$f_{0}(500)$ or $\sigma$ & $523 \pm 12$ & $518 \pm 14$ & -++ \\
\hline$f_{0}(980)$ & $991 \pm 3$ & $71 \pm 14$ & -++ \\
\hline & $1406 \pm 19$ & $160 \pm 12$ & --- \\
$f_{0}(1400)$ & $1447 \pm 27$ & $108 \pm 46$ & --+ \\
\hline
\end{tabular}

Ending this chapter let us remark that the formula (37) can be used to approximate the meson-meson transition amplitudes in different reactions where meson pairs appear. This would be the case in the meson production processes by hadrons, leptons and photons, in $\gamma \gamma$ reactions or in the central $p p$ production, in heavy particle decays, in baryon-antibaryon annihilation and so on. 


\section{Summary}

We have presented two new solutions $\mathrm{E}$ and $\mathrm{F}$ resulting from fits to experimental data on $\pi \pi$ and $K \bar{K}$ phase shifts and inelasticities. Both new solutions are characterized by the existence of a $K \bar{K}$ bound state when the interchannel couplings are switched off. In the previously found solutions $\mathrm{A}$ and $\mathrm{B}$ the $K \bar{K}$ pair remained unbound in the uncoupled case (see Table 2). In Tables 3 to 6 we have given for each solution the set of S-matrix poles lying on different sheets. Knowledge of their positions is necessary in order to evaluate masses, widths, branching ratios and coupling constants of scalar resonances studied here. We furthermore stress that not only poles of the S-matrix but also its zeroes play a very important role in evaluation of the resonance parameters. All zeroes are dynamically related. Also the knowledge of the pole trajectories as function of the interchannel coupling strengths is important to find origin of resonances.

We have paid a special attention to the phenomenological analysis of the energy region near $1400 \mathrm{MeV}$ where new scalar resonances appear [1]. A simple model of the S-matrix in three channels: $\pi \pi, K \bar{K}$ and $4 \pi$ represented by an effective $\sigma \sigma$ has been constructed ((7) to (9) and (37)). This parametrization could be used to represent phenomenologically the meson-meson amplitudes in different reactions. The model is based on the knowledge of the pole on sheet - - - common to all three channels and the zero which is specific for each channel. The diagonal $S$-matrix elements depend on three complex parameters (positions of the pole and of the zero, and the strength). This is in contrast to the Breit-Wigner formalism where only three real parameters describe resonance amplitudes. The Breit-Wigner approach has limited applicability since it restricts too much the positions of zeroes and thus it does not take fully into account the interchannel dynamics.

Branching ratios for different channels were defined and calculated for two- and three- channel models. We have studied the energy regions of the $f_{0}(980)$ and $f_{0}(1400)$ resonances and the region between them. We have noticed that different definitions of branching ratios were used in literature which lead to some confusion in past. The result is that there are no data on the $\pi \pi$ and $K \bar{K}$ branching ratios of $f_{0}(980)$ and the incorrect determinations of them were no more given in the new edition of the Particle Data Tables [1].

Above the third threshold the branching ratios form a $3 \times 3$ matrix. Each row of this matrix describes three branching ratios in the particular channel, but only two of them are independent quantities (25). Phenomenological information contained in such matrix is much richer than in the Breit-Wigner approach where only three branching ratios can be used. We have studied energy interval $1350 \mathrm{MeV}$ to 1500 $\mathrm{MeV}$ and found small branching ratios corresponding to the transitions from the $\pi \pi$ to $K \bar{K}$ channel and vice versa. This is in qualitative agreement with findings of the Crystal Barrel Group 28, 29].

We have given formulae (33), (35) and (36) for the coupling constants in the three-channel model and its different approximations. Calculated values of the cou- 
pling constants in the full model are reported in Tables 8 and 9. The $f_{0}(980)$ couples strongly to $K \bar{K}$ and the coupling of $f_{0}(1400)$ is much stronger for the $\pi \pi$ channel than for the $K \bar{K}$ channel. One should point out that the knowledge of the resonance mass and width and the coupling constants is not sufficient to give a good phenomenological description of the meson-meson dynamics.

We found that close to the $\sigma \sigma$ threshold there are two $f_{0}(1400)$ poles on the --- and --+ sheets (Table 10). This fact could be related to the presence of two scalar resonances $f_{0}(1370)$ and $f_{0}(1500)$ found in several phenomenological analyses [1]. Our model is also sufficiently flexible to accommodate scalar mesons at energies higher than $1500 \mathrm{MeV}$, like the $f_{J}(1710)$ (see Table E).

In the present version of our model the mass of the third channel is introduced as a free parameter. It is possible to construct a more involved model where one smears out this mass over a rather broad range according to the observed invariant $\pi \pi$ mass distribution. Our model of meson-meson interactions can be extended to describe more than three coupled channels (like $\eta \eta, \eta \eta^{\prime}$ and so on). In such case, however, we need more data of good precision in order to fit the parameters and to discriminate between possible solutions. Crossing symmetry constraints, if implemented, might also lead to reduce the number of parameters. Evidently new measurements should be performed near different meson-meson thresholds since even at the $K \bar{K}$ threshold the present data do not allow to obtain the $f_{0}(980)$ branching ratios. New data would help us to test different models of inter-meson interactions. It will lead us to a more profound knowledge of the structure of scalar mesons.

\section{Acknowledgments}

We would like to thank Krzysztof Rybicki for very useful discussions. This work has been performed in the framework of the IN2P3-Polish laboratories Convention (project No 93-71).

\section{Appendix A}

Here we give the full formula for the Jost function $D \equiv D\left(k_{1}, k_{2}, k_{3}\right)$ of our unitary model built to describe the three channels: $\pi \pi, K \bar{K}$ and the effective $2 \pi 2 \pi$ $(\sigma \sigma)$. Using the same notation as in $(\mathbb{[}])$ we have

$$
D=d_{0} D_{0}+\lambda_{00} I_{01} D_{1}-\lambda_{02} I_{22} D_{2}-\lambda_{03} I_{33} D_{3},
$$

where

$$
\begin{aligned}
d_{i} & =1-\lambda_{i i} I_{i i}, \quad i=0,1,2,3, \\
D_{0} & =\widetilde{D_{0}}-\lambda_{02} I_{01} I_{22} C_{2}-\lambda_{03} I_{01} I_{33} C_{3}, \\
C_{2} & =\lambda_{12}+I_{33}\left(\lambda_{13} \lambda_{23}-\lambda_{12} \lambda_{33}\right), \\
C_{3} & =\lambda_{13}+I_{22}\left(\lambda_{12} \lambda_{23}-\lambda_{13} \lambda_{22}\right),
\end{aligned}
$$




$$
\begin{aligned}
\widetilde{D_{0}}= & d_{1} d_{2} d_{3}-2 \lambda_{12} \lambda_{13} \lambda_{23} I_{11} I_{22} I_{33} \\
& -\lambda_{13}^{2} I_{11} I_{33} d_{2}-\lambda_{12}^{2} I_{11} I_{22} d_{3}-\lambda_{23}^{2} I_{22} I_{33} d_{1}, \\
D_{1}= & -I_{01} d_{2} C_{1}+\lambda_{23}\left(\lambda_{11} \lambda_{23}-\lambda_{12} \lambda_{13}\right) I_{01} I_{22} I_{33} \\
& -\left(\lambda_{02} I_{00}+\lambda_{12} I_{01}\right) I_{22} C_{2}-\lambda_{03} I_{00} I_{33} C_{3}, \\
C_{1}= & \lambda_{11}+I_{33}\left(\lambda_{13}^{2}-\lambda_{11} \lambda_{33}\right), \\
D_{i}= & \lambda_{0 i}\left\{I_{01}^{2}\left[\lambda_{11}+I_{j j}\left(\lambda_{1 j}^{2}-\lambda_{11} \lambda_{j j}\right)\right]+I_{00}\left[d_{1} d_{j}-\lambda_{1 j}^{2} I_{11} I_{j j}\right]\right\} \\
& +\lambda_{0 j} I_{j j}\left[\lambda_{23} I_{00} d_{1}+\lambda_{11} \lambda_{23} I_{01}^{2}+\lambda_{12} \lambda_{13}\left(I_{00} I_{11}-I_{01}^{2}\right)\right] \\
& +\lambda_{1 i} I_{01} d_{j}+\lambda_{1 j} \lambda_{23} I_{01} I_{j j},
\end{aligned}
$$

where $i=2,3, j=3$ if $i=2$ and $j=2$ if $i=3$.

\section{Appendix B}

We derive an approximated formula for the pion-pion $S$-matrix element given by (21). At first let us make an expansion of the Jost function in the denominator of $S_{11}$ near its zero on sheet --- at $\mathbf{k}_{d}=\left(k_{1 d}, k_{2 d}, k_{3 d}\right)$ :

$$
D(\mathbf{k}) \equiv D\left(k_{1}, k_{2}, k_{3}\right) \approx\left(k_{1}-k_{1 d}\right) d_{1}+\frac{1}{2}\left(k_{1}-k_{1 d}\right)^{2} s_{1} .
$$

Here

$$
d_{1}=\left[\frac{\partial D\left(k_{1}, k_{2}, k_{3}\right)}{\partial k_{1}}+\frac{k_{1 d}}{k_{2 d}} \frac{\partial D\left(k_{1}, k_{2}, k_{3}\right)}{\partial k_{2}}+\frac{k_{1 d}}{k_{3 d}} \frac{\partial D\left(k_{1}, k_{2}, k_{3}\right)}{\partial k_{3}}\right]_{\mathbf{k}=\mathbf{k}_{d}}
$$

and

$$
\begin{aligned}
s_{1} & =\left[\frac{\partial^{2} D\left(k_{1}, k_{2}, k_{3}\right)}{\partial k_{1}^{2}}+\frac{k_{1 d}^{2}}{k_{2 d}{ }^{2}} \frac{\partial^{2} D\left(k_{1}, k_{2}, k_{3}\right)}{\partial k_{2}^{2}}+\frac{k_{1 d}{ }^{2}}{k_{3 d}{ }^{2}} \frac{\partial^{2} D\left(k_{1}, k_{2}, k_{3}\right)}{\partial k_{3}^{2}}\right. \\
& \left.+2 \frac{k_{1 d}}{k_{2 d}} \frac{\partial^{2} D}{\partial k_{1} k_{2}}+2 \frac{k_{1 d}}{k_{3 d}} \frac{\partial^{2} D}{\partial k_{1} k_{3}}+2 \frac{k_{1 d}^{2}}{k_{2 d} k_{3 d}} \frac{\partial^{2} D}{\partial k_{2} k_{3}}\right]_{\mathbf{k}=\mathbf{k}_{d}} .
\end{aligned}
$$

In (B2) and (B3) we have used relations

$$
\begin{aligned}
& k_{2}-k_{2 d} \approx\left(k_{1}-k_{1 d}\right) \frac{k_{1 d}}{k_{2 d}}, \\
& k_{3}-k_{3 d} \approx\left(k_{1}-k_{1 d}\right) \frac{k_{1 d}}{k_{3 d}},
\end{aligned}
$$

which follow from the energy conservation (44). Similarly we make a power expansion of the Jost function in the numerator of $S_{11}$. 
Let us denote by $\mathbf{k}_{n}=\left(k_{1 n}, k_{2 n}, k_{3 n}\right)$ a zero of $D\left(k_{1}, k_{2}, k_{3}\right)$ on sheet -++ :

$$
D\left(k_{1 n}, k_{2 n}, k_{3 n}\right)=0 .
$$

Then using (四) we expand

$$
D\left(-k_{1}, k_{2}, k_{3}\right) \approx\left(k_{1}-k_{1 n}{ }^{*}\right) c_{1}+\frac{1}{2}\left(k_{1}-k_{1 n}{ }^{*}\right)^{2} t_{1},
$$

where

$$
\begin{aligned}
c_{1} & =\left[-\frac{\partial D\left(-k_{1}, k_{2}, k_{3}\right)}{\partial k_{1}}-\frac{k_{1 n}}{k_{2 n}} \frac{\partial D\left(-k_{1}, k_{2}, k_{3}\right)}{\partial k_{2}}\right. \\
& \left.-\frac{k_{1 n}}{k_{3 n}} \frac{\partial D\left(-k_{1}, k_{2}, k_{3}\right)}{\partial k_{3}}\right]_{\mathbf{k}=-\mathbf{k}_{\mathbf{n}}^{*}}
\end{aligned}
$$

and $t_{1}$ is given by an equation similar to that for $s_{1}$ in (B3).

With the help of (B1) and (B7) the pion-pion $S$-matrix element reads

$$
S_{11} \approx \frac{k_{1}-k_{1 n}^{*}}{k_{1}-k_{1 d}} f_{1}
$$

where

$$
\begin{gathered}
f_{1}=\frac{c_{1}}{d_{1}} F\left(k_{1}\right), \\
F\left(k_{1}\right)=\frac{1+\frac{1}{2} \frac{t_{1}}{c_{1}}\left(k_{1}-k_{1 n}{ }^{*}\right)}{1+\frac{1}{2} \frac{s_{1}}{d_{1}}\left(k_{1}-k_{1 d}\right)} .
\end{gathered}
$$

The function $F\left(k_{1}\right)$ can further be approximated by a constant if we limit ourselves to values $k_{1}$ close to $R e k_{1 d}$ in the denominator of $F\left(k_{1}\right)$ and close to $R e k_{1 n}$ in its numerator:

$$
F\left(k_{1}\right) \approx \frac{1+\frac{1}{2} \frac{t_{1}}{c_{1}} i \operatorname{Im} k_{1 n}}{1-\frac{1}{2} \frac{s_{1}}{d_{1}} i \operatorname{Im} k_{1 d}} .
$$

Similar expressions can be derived for the diagonal $K \bar{K}$ and $\sigma \sigma S$-matrix elements.

\section{References}

[1] Particle Data Group, C. Caso et al., Europ. Phys. J. C3 (1998) 1.

[2] Proceedings of the Hadron Spectroscopy Conference, Upton, NY, August 1997, Editors S.-U. Chung and H. J. Willutzki, American Institute of Physics, Woodbury, New York (1998). 
[3] F. E. Close, G. R. Farrar and Z. Li, Phys. Rev. D55 (1997) 5749.

[4] W. Lee and D. Weingarten, Nucl. Phys. B (Proc. Supl.) 63A-C (1998) 194.

[5] C. Michael, see [2] p. 657.

[6] F. E. Close, Nucl. Phys. B (Proc. Supl.) 63A-C (1998) 28.

[7] R. Kamiński, L. Leśniak and B. Loiseau, Phys. Lett. B413 (1997) 130.

[8] R. Kamiński, L. Leśniak, and K. Rybicki, Z. Phys C74 (1997) 79.

[9] R. Kamiński, L. Leśniak and J.-P. Maillet, Phys. Rev. D50 (1994) 3145.

[10] A. Abele et al., Phys. Lett. B380 (1996) 453.

[11] N. N. Achasov and G. N. Shestakov, Phys. Rev. D58 (1998) 054011.

[12] L. Rosselet et al., Phys. Rev. D15 (1977) 574.

[13] A.A. Belkov et al., Pisma Zh. Eksp. Teor. Fiz. 29 (1979) 652.

[14] V. Srinivasan et al., Phys. Rev. D12 (1975) 681.

[15] D. Cohen et al., Phys. Rev. D22 (1980) 2595.

[16] G. Grayer et al., Nucl. Phys. B75 (1974) 189.

[17] H. Becker et al., Nucl. Phys. B151 (1979) 46.

[18] W. M. Kloet and B. Loiseau, Eur. Phys. J. A 1 (1998) 337.

[19] L. Leśniak, Acta Phys. Pol. B27 (1996) 1835.

[20] Particle Data Group, Phys. Rev. D 45, part II (1992); Particle Data Group, Phys. Rev. D50, part I (1994).

[21] P. F. Loverre et al., Z. Phys. C6 (1980) 187.

[22] W. Wetzel et al., Nucl. Phys. B115 (1976) 208.

[23] N. M. Cason et al., Phys. Rev. 41 (1978) 271.

[24] D. Morgan and M.R. Pennington, Phys. Rev. D48 (1993) 1185.

[25] D. V. Bugg, A. V. Sarantsev and B. S. Zou, Nucl. Phys. B471 (1996) 59.

[26] F. Cannata, J.-P. Dedonder, and L. Leśniak, Phys. Lett. B207 (1988) 115.

[27] L. Goerlich et al. (CERN-Cracow-Munich Collaboration), Nucl. Phys. B174 (1980)16. 
[28] C. Amsler, hep-ex/9708025, to be published in Rev. Mod. Phys.

[29] A. Abele et al., Phys. Rev. D57 (1998) 3860.

[30] A. Abele et al., Nucl. Phys. A609 (1996) 562. 